\title{
Viertes Kapitel: Die Praxis der Wiedergutmachung in der US-Zone (1949-1954)
}

\section{Durchführung des Entschädigungsgesetzes}

\section{Entschädigung auf Raten}

Vier Jahre nach dem Ende des nationalsozialistischen Regimes bestand also in den Ländern der amerikanischen Besatzungszone endlich ein Gesetz, das rassisch, religiös und politisch Verfolgten einen Rechtsanspruch auf Entschädigung ihrer Schäden an Leben, Körper, Gesundheit, Freiheit, Eigentum, Vermögen oder im wirtschaftlichen Fortkommen einräumte ${ }^{1}$. Es löste zugleich das Sonderfondsgesetz $\mathrm{ab}$, bei dem es sich im Grunde nur um eine erweiterte Fürsorgeregelung für Verfolgte des Nationalsozialismus gehandelt hatte, da die Leistungen von der Bedürftigkeit der Antragsteller abhängig waren ${ }^{2}$. Bis zum Sommer 1949 wurden im Rahmen des Sonderfondsgesetzes ca. 42,7 Mio. DM ausgezahlt, davon etwa 17,5 Mio. in Bayern, 13,9 Mio. in Hessen, 5,7 Mio. in Württemberg-Baden und 5,7 Mio. in Bremen ${ }^{3}$.

Ende 1952 lagen rund 275.000 Anmeldungen nach dem Entschädigungsgesetz der US-Zone vor, davon allein ca. 177.000 in Bayern, 40.000 in Hessen, 46.000 in Württemberg-Baden und 12.000 in Bremen. Augenfällig ist, daß mit knapp zwei Dritteln der Anträge Bayern die Hauptlast zu tragen hatte, was hauptsächlich mit der Massierung der DP's in diesem Land zusammenhing. Dort hielten sich nach Kriegsende teilweise bis zu 130.000 jüdische und andere DP's auf ${ }^{4}$, die als eine schwere wirtschaftliche und soziale Belastung angesehen wurden, mußten doch auf dem Wege über die Besatzungskosten erhebliche Beträge für diese Gruppe aufgebracht werden ${ }^{5}$.

Doch bis zur Realisierung der Entschädigungsansprüche war es meist ein langer und oft auch mühseliger Weg. Ende 1951 faßte Eli W. Debevoise, der Rechtsberater des amerikanischen Hohen Kommissars, vor dem Foreign Affairs Committee des USKongresses, das sich zu einem Informationsbesuch in Deutschland befand, die Situation bei der Durchführung des Entschädigungsgesetzes in der US-Zone treffend zusammen: „The four Laender (states) in the American Zone have got pretty good laws and they

${ }^{1} \$ 1$, Gesetz zur Wiedergutmachung nationalsozialistischen Unrechts vom 12.8. 1949 (USEG), in: Bayerisches Gesetz- und Verordnungsblatt (BayGVOBl), Nr. 20, 29. 8. 1949, S. 195-204.

2 Vgl. Drittes Kapitel, Abschnitt III. 1.

${ }^{3}$ Report of Survey of General Claims Activities in the US Zone as of March 31, 1950, Office of the U.S. High Commissioner for Germany, Office of Economic Affairs, Property Division, IfZ-Archiv, MF 260, OMGUSCAD, 17/256-2/23.

4 Schlußbericht des Untersuchungsausschusses zur Prüfung der Vorgänge im Landesentschädigungsamt (LEA) v. 10.12. 1953, Bayerischer Landtag, 2. Wahlperiode, Drucksachen, Beilage 5128, S. 4.

5 Vgl. dazu Institut für Besatzungsfragen, Das DP-Problem. Eine Studie über die ausländischen Flüchtlinge in Deutschland, Tübingen 1950, S. 87-89. Demnach hätten allein die US-Zonen-Länder von 1945 bis März 1949 etwa 800 Mio. RM bzw. DM für die DP's aufgebracht, für alle drei westlichen Zonen zusammen habe der entsprechende Betrag bei ca. zwei Mrd. RM bzw. DM gelegen. 
are functioning fairly well. They would function better if the Laender had more money. "6 Die zweifelhafte Finanzierungsgrundlage des Entschädigungsgesetzes der USZone drohte nun die guten Absichten dieser Regelung ad absurdum zu führen.

Die Länder hatten den Finanzierungsschwierigkeiten in erster Linie dadurch Rechnung getragen, daß sie die dort festgelegten Ansprüche in drei Klassen eingeteilt und mit unterschiedlichen Erledigungsfristen versehen hatten. Insgesamt mußten die Leistungen so erst bis zum Jahr 1960 erbracht sein ${ }^{7}$. Hessen, Württemberg-Baden und Bremen finanzierten die Entschädigungszahlungen aus dem Staatshaushalt, während Bayern hier zunächst mit den Mitteln einer „Stiftung zur Wiedergutmachung nationalsozialistischen Unrechts" auszukommen hoffte. Hierin waren Werte aus nationalsozialistischen Konzentrationslagern, Sühneabgaben aus der Entnazifizierung etc. gesammelt. In gewisser Weise lebte darin noch der Gedanke fort, daß die Finanzierung der Wiedergutmachung nicht von der Gesamtheit des deutschen Volkes - sprich Steuerzahler - getragen werden sollte, sondern sich vor allem auf das Vermögen nationalsozialistischer Organisationen und Nutznießer stützen müsse. Allerdings hatte sich der Exklusivanspruch der Verfolgten des Nationalsozialismus auf diese Werte immer deutlicher zu einem sehr zweifelhaften Privileg entwickelt, da Mittel dieser Herkunft offensichtlich nur in geringem Umfang aufgebracht werden konnten.

Leistungen nach Klasse II und III des Entschädigungsgesetzes sollte es überdies nur geben, „sofern und soweit die hierzu erforderlichen Deckungsmittel aus dem Lastenausgleich zur Verfügung gestellt werden “ ${ }^{8}$. Doch war es von vornherein unwahrscheinlich, daß aus dieser Quelle tatsächlich irgendwelche Mittel fließen würden. Bereits im Zusammenhang des Soforthilfegesetzes waren entsprechende Bemühungen erfolgslos geblieben. Und am 15. September sowie erneut am 1. Dezember 1950 lehnte auch das Bundeskabinett einen durch Philipp Auerbach namens des Koordinierungsbüros der interministeriellen Arbeitsgemeinschaft für Wiedergutmachungs- und Entschädigungsfragen gestellten Antrag ab, zehn Prozent des Aufkommens aus dem Lastenausgleich zur Erfüllung der bestehenden Entschädigungsgesetze der Länder zugunsten der Verfolgten des Nationalsozialismus zur Verfügung zu stellen'. Angesichts dieser Lage forderte McCloy die Ministerpräsidenten der US-Zone am 9. April dringend dazu auf, sich über alternative Finanzierungsquellen Gedanken zu machen ${ }^{10}$.

Dennoch hielten die Länder zunächst noch einige Zeit an der Fiktion fest, auf diesem Wege ihre Finanzierungsprobleme lösen zu können. Während aber Württemberg-Baden bereit war, die Entschädigung nötigenfalls auch aus Haushaltsmitteln zu bezahlen ${ }^{11}$, versuchte Bayern die entstehende Finanzierungslücke durch Kredite zu schlie-

${ }^{6}$ Office of the U.S. High Commissioner for Germany, Stenographic Record of the Briefing Sessions, November 12, 1951, Headquarters Building, Frankfurt, Transcript of Briefing for the Special Sub-Committee of the House Foreign Affairs Committee (German Study Mission), S. 8, Washington National Record Center, Suitland, (WNRC), RG 466, Records of the U.S. High Commissioner for Germany (McCloy Papers), Box 33.

\ 38 , USEG.

8 \$9, ebenda.

9 96. Kabinettssitzung am 15.9 und 114. Kabinettssitzung am 1.12. 1950, in: Die Kabinettsprotokolle der Bundesregierung, hrsg. f. d. Bundesarchiv v. Hans Booms, Bd. 2: 1950, bearb. v. Ulrich Enders u. Konrad Reiser, Boppard a.Rh. 1984, S.694 u. 852.

10 John J. McCloy an Reinhold Maier, 9. 4. 1951, BadWürtHStA, EA 1/920, Bü. 709; ders. an Hans Ehard, 11. 4. 1951, BayHStA, MA 114240. Vgl. dazu auch William G. Daniels an Alex F. Kiefer (State Department/ Property Division), 24.3. 1951, USNA, RG 59, 262.0041/3-2451.

11 R. Maier an McCloy, 19.4. 1951, BadWürtHStA, EA 1/920, Bü. 709. 
ßen ${ }^{12}$. Dies hatte zur Folge, daß im Sommer 1951, als in Bayern monatlich gerade eine Million DM für das Entschädigungsgesetz bereitstanden, allein 350.000 DM Zinsen gezahlt werden mußten ${ }^{13}$. Doch bald darauf ging man notgedrungen auch hier endgültig dazu über, die Entschädigung aus dem Staatshaushalt zu finanzieren, während die Stiftung zur Wiedergutmachung nationalsozialistischen Unrechts aufgelöst wurde ${ }^{14}$.

Damit hatte die Entwicklung, die Finanzierung der Wiedergutmachung zu einer ordentlichen Haushaltsaufgabe zu machen, ihren Abschluß gefunden. Die ursprünglich vorhandene Verknüpfung mit dem Sühnegedanken war dabei endgültig entfallen. Die Finanzierung der Wiedergutmachung auf diesem Wege hatte sich als utopisch erwiesen, zumal die mit diesem Modell verknüpften gesellschaftspolitischen Prämissen inzwischen obsolet waren. Die Entschädigung geriet dadurch natürlich in direkte Konkurrenz zu allen anderen Haushaltsausgaben. Doch auf lange Sicht bildete dies auch die Voraussetzung dafür, daß die erheblichen Summen, die bis heute für diesen Zweck in der Bundesrepublik aufgebracht wurden, überhaupt zur Verfügung gestellt werden konnten. Mit der Übernahme der Finanzierung der Entschädigung auf die öffentlichen Haushalte wurde zwar keine Kollektivschuld anerkannt, aber immerhin einer „Kollektivverantwortung" Rechnung getragen.

Alles in allem zeigte sich Bayern, wie in Washington aufmerksam registriert wurde, in dieser Zeit am wenigsten in der Lage, den finanziellen Anforderungen des Entschädigungsgesetzes gerecht zur werden, was aber auch damit zu tun hatte, daß hier, wie gesagt, mit Abstand die meisten Ansprüche gestellt wurden. Vor allem die große Zahl jüdischer DP's in Bayern schuf auch in dieser Hinsicht enorme Probleme. Nur widerwillig hatten die US-Zonen-Länder die Verpflichtung zur Entschädigung dieser Gruppe übernommen. Wie Ministerpräsident Ehard im November 1951 Bundeskanzler Adenauer mitteilte, waren die Länder „der übereinstimmenden Auffassung, daß die Entschädigung der DP's eine Angelegenheit des Bundes“ ${ }^{15}$ sei. Dies war noch höflich ausgedrückt. Der deutsche Generalkonsul in London, Max Bachmann, berichtete Herbert Blankenhorn Anfang 1952 über die dort herrschende Unzufriedenheit wegen der Abwicklung der Entschädigung in Bayern und klagte bei dieser Gelegenheit, daß „in höchsten bayerischen Regierungskreisen die Auffassung herrscht, wenn der Herr Bundeskanzler mit der jüdischen Wiedergutmachung Politik treiben will, so müssen die Mittel dafür von Seiten der Bundesregierung aufgebracht werden “16.

Für die Verfolgten hieß dies nun, daß sie darauf warten mußten, bis ihre Entschädigungsforderungen sukzessive befriedigt wurden. Erst entsprechende Durchführungsverordnungen der einzelnen Länder schufen jeweils die Voraussetzungen dafür, daß Anträge bestimmter Anspruchsgruppen überhaupt bearbeitet werden konnten. Wegen der unterschiedlichen Relation zwischen der jeweiligen Zahl der Ansprüche und den zur Verfügung stehenden Mitteln, wegen wechselnder Prioritäten und aus anderen Gründen verlief dieser Prozeß in den einzelnen Ländern der US-Zone nicht einheitlich: Unterschiede ergaben sich sowohl im Bearbeitungstempo als auch in der Reihenfolge der Befriedigung bestimmter Ansprüche. Überdies wurde die Arbeit an der Durchfüh-

\footnotetext{
12 Ehard an McCloy, 7.6. 1951, BayHStA, MA 114240.

13 Jack K. McFall (Assistant Secretary of State) an Senator Lehmann, 29. 8. 1951, USNA, RG 59, 262.0041/62951.

14 Bayerischer Ministerrat, 24. 11. 1953, IfZ-Archiv, NL Hoegner, ED 120, Bd. 378.

15 Ehard an Konrad Adenauer, 23. 11. 1951, PA/AA, II 244-13 II, Bd. 6.

16 Max Bachmann an Herbert Blankenhorn, 11. 1. 1952, PA/AA, II 244-11 II, Bd. 1.
} 
rung des Entschädigungsgesetzes der US-Zone im Herbst 1953 durch den Erlaß des Bundesentschädigungsgesetzes unterbrochen.

Mit welchen Schwierigkeiten die Verfolgten bei der Einlösung ihrer Ansprüche nach dem Entschädigungsgesetz zu rechnen hatten, kann am Beispiel der Haftentschädigung besonders gut gezeigt werden. Für viele ehemalige KZ-Häftlinge war dies der einzige Entschädigungsanspruch, den sie besaßen. Die erste Hälfte der Haftentschädigung bis zum Betrag von 3.000 DM war in die erste Rangklasse eingeteilt und wurde deshalb nach Erlaß der Haftentschädigungsverordnungen fällig ${ }^{17}$. Doch forderte insbesondere die VVN die baldige Auszahlung auch der zweiten Rate der Haftentschädigung und protestierte deshalb wiederholt gegen die „Verschleppung ihrer Ansprüche ${ }^{{ }^{18}}$. Staatssekretär Ringelmann aus dem bayerischen Finanzministerium wehrte dies damit ab, „daß eine große Zahl von Mitgliedern der VVN Kommunisten sind, die als solche im wesentlichen nur Haftentschädigungsansprüche geltend machen konnten." Eine raschere Durchführung der Wiedergutmachung ließe sich zudem „schon aus finanziellen Gründen nicht bewerkstelligen und ... (sci. sei) auch im Entschädigungsgesetz gar nicht vorgesehen ${ }^{19}$. Hier wurde deutlich, daß die politische Auseinandersetzung mit den Kommunisten die Entschädigung immer stärker belastete: Noch wurden Ansprüche kommunistischer Verfolgter nicht generell in Frage gestellt, doch wertete Ringelmann die „zahlreichen Eingaben und unerfüllbaren Forderungen“ der VVN in erster Linie als einen Versuch, „Unruhe in die Kreise der Verfolgten zu tragen und die Autorität der Staatsregierung zu untergraben ${ }^{20}$.

Württemberg-Baden schlug deshalb den Weg ein, die zweite Rate der Haftentschädigung vorzeitig auszuzahlen, sofern diese für den sozialen Wohnungsbau verwendet würde und so nicht "dem wilden Verbrauch oder undurchsichtigen Zwecken" anheimfiele und insbesondere nicht etwa „zur Propagierung einer weiteren Radikalisierung Verwendung " fände ${ }^{21}$. Das schwäbische Modell - frei nach dem Motto: Häusle bauen statt revoluzzern - machte schließlich auch in Hessen Schule 22 .

Eine andere Methode, die Abwicklung der Entschädigung zu beschleunigen, war der Vergleich. In Bayern erhielten die Antragsteller einen sogenannten Feststellungsbescheid, in dem die Gesamthöhe ihres Haftentschädigungsanspruchs festgestellt wur$\mathrm{de}^{23}$. Während die erste Rate bis zu einer Höhe von 3.000 DM innerhalb eines Monats ausgezahlt wurde, sollte die zweite Rate nach Maßgabe vorhandener Deckungsmittel bis zum 31. März 1954 zur Auszahlung gelangen. Mit den Feststellungsbescheiden entwickelte sich ein schwunghafter Schwarzhandel, wobei Geschäftemacher diese den Verfolgten häufig zu einer Rate von nur 20 Prozent des Nominalwertes abkauften, um sie dann zu gegebener Zeit in voller Höhe einzulösen.

Deshalb tüftelten der Präsident des Landesentschädigungsamts Auerbach und das

$17 \$ 38$ Abs. 6, USEG; 1.Verordnung zur Durchführung des Entschädigungsgesetzes (Haftentschädigungsverordnung) vom 28.11. 1949, in: BayGVOBl, Nr. 28, 10. 12. 1949, S. 287-289.

18 Siehe etwa Stellungnahme des Landesvorstands der VVN Hessen, 31.3. 1950, HessHStA, Abt. 502, Nr. 2773a; Carola Carg (VVN Bayern) an Ministerpräsident Ehard, 29. 7. 1950, BayHStA, MA 114240.

19 Richard Ringelmann an bayerische Staatskanzlei, 10.11. 1950, BayHStA, MA 114240.

20 Ebenda.

21 Wolfgang Haußmann (Vorsitzender des Wirtschafts- u. Verkehrsausschusses des Landtags von Württemberg-Baden) an das Staatsministerium, 19.10. 1949, BadWürtHStA, EA 1/920, Bü. 709.

${ }^{22}$ Kabinettsvorlage vom 19.3. 1951, HessHStA, Abt. 502, Nr. 2013.

${ }^{23} \$ 9$ Abs.1, 1, Haftentschädigungsordnung. 
bayerische Finanzministerium mit dem Segen des Ministerpräsidenten Ehard ein Projekt aus, wonach die Haftentschädigungsfestsetzungsbescheide auf dem Wege über eine Sammelstelle zu einem Kurs von 47 Prozent des Nominalwertes durch ein Bankenkonsortium aufgekauft werden sollten. Zugleich verpflichtete sich der bayerische Staat, die aufgekauften Feststellungsbescheide spätestens zwei Jahre nach Erwerb zum Kurs von 62 Prozent des Nennwertes einzulösen ${ }^{24}$. Auf diese Weise sparte der bayerische Staat ca. 40 Prozent der fälligen Beträge; wichtiger war aber noch die hierdurch eintretende Beschleunigung der Auswanderung jüdischer DP's, da diese ihre Abreise häufig vom Erhalt ihrer Entschädigungszahlungen abhängig machten. So mußten die Entschädigungsberechtigten nachweisen, daß sie die zweite Rate vorzeitig zum Behufe der Auswanderung bzw. zur Begründung einer Existenz und ähnlichen Zwecken benötigten.

Auerbach versuchte diesen Weg auch der hessischen Regierung schmackhaft zu machen, doch kamen hier Bedenken gegen einen derartigen Handel auf. Der hessische Finanzminister Werner Hilpert witterte die Gefahr, daß die ausländische Presse dies als eine „zweite Judenvermögensabgabe“ brandmarken könnte sowie daß die Berechtigten diese ungleichen Verträge später anfechten könnten ${ }^{25}$. Württemberg-Baden hatte hingegen bereits eine eigene Variante dieser Praxis entwickelt: Hier erhielten die Geschädigten 60 Prozent des Nominalwertes ihrer zweiten Rate ausbezahlt, sofern sie auf alle übrigen Ansprüche nach dem Entschädigungsgesetz verzichteten ${ }^{26}$. Aus Praktiken dieser Art entwickelte sich ein erheblicher Sumpf halblegaler und illegaler Geschäfte um die Entschädigung, die für das Bild der Wiedergutmachung in der öffentlichen Meinung ausgesprochen nachteilig waren. Davon wird später noch ausführlicher die Rede sein.

\section{Die Ausgrenzung von Verfolgten durch Rechtsprechung und Verwaltung}

Die Finanzierungsschwierigkeiten führten aber nicht nur zur Streckung der Zahlungen, sondern im Prozeß der Implementierung des Entschädigungsgesetzes durch Verwaltung und Gerichte auch zu einer weiteren Einschränkung des Berechtigtenkreises. Wiederholt stützte die Rechtsprechung ablehnende Urteile damit, „daß schon im Hinblick auf die Beschränktheit der verfügbaren Mittel von vornherein nicht daran gedacht werden konnte, die Fülle natsoz. Unrechts im vollen Umfang wiedergutzumachen, sondern unter den Opfern der natsoz. Verfolgung nach gewissen Gesichtspunkten, hauptsächlich der Würdigkeit, eine gewisse Auswahl getroffen werden mußte" ${ }^{27}$. Auf diesem Wege wurde auch der Grundsatz, daß nicht allein die Schwere der erlittenen Verfolgung, sondern nur der Zusammenhang mit spezifischen Verfolgungsgründen zu einer Entschädigung berechtige, weiter verstärkt. Hierbei handelt es sich wohl um den Punkt, der aus heutiger Perspektive am meisten Schwierigkeiten bei der Bewertung der Strukturen der Entschädigung für Opfer des Nationalsozialismus bereitet.

24 Philipp Auerbach an Ministerpräsident Georg August Zinn, 12. 12. 1950, HessHStA, Abt. 502, Nr. 2772c; Anklageschrift der 1. Strafkammer des Landgerichts München I, Prozeß gegen Auerbach und drei Andere, S. 66-69, Landgericht München I, Az. 2 KLs 1/52, Bd. I.

25 Werner Hilpert an Zinn, 22. 12. 1950, HessHStA, Abt. 502, Nr. 2772c.

${ }^{26}$ Anklageschrift der 1.Strafkammer des Landgerichts München I, Prozeß gegen Auerbach und drei Andere, S. 89 (Anm. 24).

27 Entschädigungskammer München I, Urteil v. 2.5. 1951, EK Nr.79/50, in: Rechtsprechung zum Wiedergutmachungsrecht (RzW) 2 (1951), S.289. Siehe auch Wiedergutmachungskammer Stuttgart, Urteil v. 20.4. 1950, ES 3782 - EGR 103, in: RzW 1 (1949/50), S. 315; Wiedergutmachungskammer Stuttgart, Urteil v. 29.9. 1950, ES 2979 - EGR 762, in: RzW 2 (1951), S. 10. 
Ganz besonders rigoros wurde dieser Grundsatz bei den ausländischen Verfolgten angewandt. Das Entschädigungsgesetz der US-Zone räumte allein rassisch, religiös oder politisch verfolgten DP's, die sich am 1. Januar 1947 in einem DP-Lager befunden hatten, einen Anspruch auf Entschädigung ein - damit blieben alle diejenigen DP's, die vor diesem Zeitpunkt ausgewandert waren, ausgeschlossen ${ }^{28}$. Doch schränkten Rechtsprechung und Verwaltung die nach dem Entschädigungsgesetz bestehenden Ansprüche weiter ein. So wurden im Prinzip nur jüdischen DP's Entschädigungsansprüche zugestanden. Bei nichtjüdischen DP's erkannte man hingegen in der Regel keine rassische, religiöse oder politische Verfolgung an, sondern reihte sie unter die sogenannten Nationalverfolgten ein, für die das Entschädigungsgesetz keine Ansprüche vorsah.

Dies zielte insbesondere auf nichtjüdische Polen. $\mathrm{Da}$ sie nicht als Juden verfolgt worden waren, urteilten die Gerichte konstant, daß ihre Verfolgung die Folge nationaler Auffassungen sei, die sich nicht gegen den Nationalsozialismus als solchen gerichtet hätten, sondern Ausdruck eines Volkstumskampfes zwischen Deutschen und Polen gewesen seien, der viel älter als der Nationalsozialismus $\operatorname{si}^{29}$. So wurde allein eine innenpolitische Gegnerschaft zum Nationalsozialismus als Verfolgungsgrund anerkannt, Widerstand gegen die deutsche Besatzung galt hingegen in aller Regel als nationale Regung gegen die Okkupanten ${ }^{30}$. Nur in Ausnahmefällen führte diese Praxis auch zur Anerkennung von Entschädigungsansprüchen etwa von Anhängern der Polnischen Sozialistischen Partei (PPS), da diese wegen ihrer demokratisch-sozialistischen Überzeugung verfolgt worden seien ${ }^{31}$.

Über die Nichtentschädigung von Nationalverfolgten nach dem Entschädigungsgesetz bestand auf deutscher Seite große Einmütigkeit. So erklärte auch Otto Küster, „daß die Grenze, die das Gesetz zieht, eben verläuft zwischen politischen Widerstandskämpfern und nationalen Widerstandskämpfern. Wir haben das ausdrücklich so ins Auge gefaßt, daß diejenigen Leute, die im Rahmen des nationalen Widerstandes gegen Deutschland verhaftet worden sind, in dieses Gesetz nicht hineingehören“. Deshalb lehnte Küster die Entschädigung der Nationalverfolgten $a^{32}$. Er beharrte auch hier auf der strengen Unterscheidung von Wiedergutmachung und Reparationen und verwies dabei die Ansprüche der Nationalverfolgten in den Bereich der letzteren: „Die Entschädigung der Opfer von Unmenschlichkeiten, die im Zuge des Nationalitätenkampfes begangen wurden, wird die Sache der Auseinandersetzung von Staat zu Staat sein. “ ${ }^{33}$ So beschlossen auch die Wiedergutmachungsreferenten der elf Länder Mitte 1950, daß nur "derjenige Ausländer, der unter den gleichen Umständen, wenn er ein Deutscher gewesen wäre, verfolgt worden wäre, Anspruch hat, nach dem Gesetz behandelt zu werden ${ }^{34}$.

Die Umsetzung in Verwaltungsrichtlinien zeigt etwa Runderlaß Nr.110 des hessischen Innenministeriums vom Juli 1952, der alle Entschädigungsbehörden kategorisch anwies, daß nur Ansprüche jüdischer DP's anerkannt werden könnten, Anträge nicht-

${ }^{28} \$ 6(1) 3$, USEG.

29 Entschädigungskammer Stuttgart, Urteil v. 5. 12. 1949 - Es 507 (3931), in: RzW 1 (1949/50), S. 125.

${ }^{30}$ OLG Frankfurt a.M., Urteil v. 20.11. 1950, 2 W 315/50, in: RzW 2 (1951), S. 93; LG München, Urteil v. 22. 11. 1950, EK 109/50, ebenda.

31 OLG Stuttgart, Urteil v. 12. 4.1950, EGR 24, in: RzW 2 (1951), S. 93.

32 Protokoll der Sitzung des Koordinierungsausschusses der 11 Länder, 25.5. 1950, S. 18, BayMJ, 1101a, H. 4.

${ }^{33}$ Vgl. Otto Küster, Anmerkung zu der Entscheidung der Entschädigungskammer Stuttgart vom 5. 12. 1949, Es 507 (3931), in: RzW 1 (1949/50), S. 125.

34 Ebenda. 
jüdischer DP's hingegen abzulehnen seien ${ }^{35}$. Dies wurde damit begründet, daß es sich bei DP's, „die sich auf eine Verfolgung wegen politischer Überzeugung usw. berufen, um Personen (handle), die sich den Anordnungen der deutschen Besatzungsmacht widersetzt oder die sich sonst gegen die deutsche Besatzungsmacht betätigt hatten bzw. von denen eine derartige Betätigung erwartet wurde. Für ihr Verhalten gegenüber der Besatzungsmacht dürften im allgemeinen nicht eine spezifisch antinationalsozialistische Überzeugung, sondern Vaterlandsliebe und $\mathrm{Haß}$ gegen den fremden Eroberer bestimmend gewesen sein. Auch die deutschen Dienststellen werden ihre Maßnahmen in aller Regel nicht, wegen' der politischen Überzeugung des Betreffenden, sondern ohne Rücksicht auf diese lediglich unter dem Gesichtspunkt der militärischen Sicherheit bzw. des Volkstumskampfes getroffen haben. ${ }^{36}$ Derartige amtliche Exkulpationen der deutschen Besatzungsherrschaft in Polen lesen sich heute wie purer Zynismus, doch reflektieren sie nur das, was zu dieser Zeit weitverbreiteter Konsens war.

Aber auch für jüdische DP's wurden zusätzliche Hürden errichtet, und zwar insbesondere für denjenigen Teil, der erst nach Kriegsende nach Deutschland gekommen war, die sogenannten „Infiltrees“. Während Bayern und Württemberg-Baden beschlossen, sich hier an den Wortlaut des Entschädigungsgesetzes zu halten und den betreffenden Personen bei Nachweis ihrer Verfolgung prinzipiell den ihnen zustehenden Anspruch einzuräumen ${ }^{37}$, verhielt sich Hessen hier anders: Dem hessischen Runderlaß Nr. 110 zufolge waren deren Ansprüche pauschal abzulehnen, denn Deutschland habe den Heimatstaaten dieser Menschen die zur Durchführung der Wiedergutmachung erforderlichen Reparationen bereits geleistet. Zusätzlich wurde dies aber auch damit begründet, es habe sich als unmöglich herausgestellt, „die Richtigkeit der Angaben dieser Gruppe von Antragstellern auch nur mit einiger Sicherheit nachzuprüfen “ ${ }^{38}$. Das war eine Reaktion auf die Entdeckung von umfangreichen Betrügereien beim Nachweis von Verfolgungen, die allerdings auch eine Folge der verheerenden Beweisnot insbesondere der aus dem Osten kommenden Verfolgten waren.

Der Grundsatz, daß nur deutsche Verfolgte eine Entschädigung erhalten sollten, von dem im Entschädigungsgesetz der US-Zone nur aufgrund des amerikanischen Drucks eine partielle Abweichung konzediert worden war, wurde also durch die Auslegung von Gerichten und Behörden weiter verstärkt. Bei dem sogenannten Territorialitätsprinzip, das auf der kategorialen Unterscheidung von Wiedergutmachung und Reparationen basiert und geschädigte Staatsbürger ausländischer Staaten auf ihre eigenen Regierungen verweist, handelt es sich um einen der wesentlichen Pfeiler des deutschen Wiedergutmachungsrechts, der bis heute nicht erschüttert wurde ${ }^{39}$.

35 Runderlaß Nr. 110 des hessischen Ministers des Innern, Abt. VI - Wiedergutmachung, gez. Dr. Schuster, an die Regierungspräsidenten und Verteiler, 1.7. 1952, S. 2, HessHStA, Abt. 502, Nr. 2772 b.

${ }^{36}$ Ebenda.

37 Küster an die Entschädigungsbehörden und Entschädigungsgerichte des Landes Württemberg-Baden, 20.8. 1951, BadWürtHStA, EA 1/90, Bü. 709. Siehe auch Mitteilungsblatt des Beirats beim Bayerischen Landesentschädigungsamt, Januar 1952, Nr.60, S.4, „Wiedergutmachungsansprüche der DP's“.

38 Runderlaß Nr. 110 des hessischen Innenministeriums vom 1.7. 1952 (Anm. 35).

39 Vgl. dazu Ulrich Herbert, Nicht entschädigungsfähig? Die Wiedergutmachungsansprüche der Ausländer, in: Wiedergutmachung in der Bundesrepublik Deutschland, hrsg. v. Ludolf Herbst u. Constantin Goschler, München 1989, S. $292 \mathrm{ff}$. 
Das Entschädigungsgesetz der US-Zone sprach demjenigen, der „wegen seiner politischen Überzeugung“ verfolgt worden war, ein Recht auf Entschädigung $\mathrm{zu}^{40}$. Hier handelte es sich sozusagen um Urgestein der Entschädigung, denn politisch Verfolgte hatten von Anfang an zum Kern der Berechtigten gezählt. Doch begannen die Gerichte alsbald, aus diesem Brocken eine Heldenstatue mit recht schmaler Silhouette herauszumeißeln: So formulierte das Oberlandesgericht Stuttgart am 17. Februar 1950 exemplarisch, daß als „politische Überzeugung ... nur eine charaktervolle, auf sittlichen Grundlagen beruhende und während einer gewissen Zeitdauer bewährte Grundeinstellung in den Fragen des Verhältnisses zwischen Staat und Einzelpersönlichkeit anerkannt werden" könne ${ }^{41}$. Auch Otto Küster unterstrich, daß man sich bei dem Entschädigungsgesetz nicht mit einer bloßen Gegnerschaft gegen den Nationalsozialismus allein habe begnügen wollen: „Diese kann momentan gewesen sein und traf leider nicht selten zusammen mit einer asozialen, auch und erst recht gegen einen Rechtsstaat sich auflehnenden Gesinnung. Es war ja gerade der Jammer der natsoz. Zeit“, so Küster, „daß so wenig vollgültige Persönlichkeiten sich zum Widerstand bereit fanden und daß der Widerstand damit weithin fragwürdigen Existenzen überlassen blieb“ “2 . Die Rechtsprechung stellte demgegenüber Maßstäbe auf, die auf die Helden eines klassischen Dramas zielten.

So genügte es nicht allein nachzuweisen, daß eine gegen den Nationalsozialismus gerichtete Tat zu einer Verfolgung geführt hatte, sondern, wie im Juni 1950 gleichfalls das Oberlandesgericht Stuttgart konkretisierte, ein politisch Verfolgter müsse auch beweisen können, „daß die Tat, wegen der er verfolgt und geschädigt wurde, eine Tat aus politischer Überzeugung war." Humanitäre Gründe für sich allein galten dabei als nicht ausreichend ${ }^{43}$. Das führte in der Praxis unter anderem dazu, daß es die Mehrzahl der Entschädigungsgerichte ablehnte, Verfolgte aus dem Kreis der „Weißen Rose“ oder des 20. Juli nach dem USEG zu entschädigen. Die Länder mußten deshalb von sich aus mit übergesetzlichen Mitteln eingreifen, um hier zu helfen ${ }^{44}$. Ein anderer Fall, der die Problematik verdeutlicht, ist der des Wehrmachtsoffiziers, der den Befehl zur Sprengung der Brücke bei Remagen nicht rechtzeitig gegeben hatte. Dieser Soldat wurde auf Befehl Hitlers hingerichtet, doch konnte man seinen Angehörigen nach dem USEG nicht helfen, da sein Handeln nicht aus „politischer Überzeugung “ erwachsen war ${ }^{45}$.

Überdies hatten es Gerichte und Entschädigungsämter häufig etwa mit Personen zu tun, die in Gaststätten oder sonstwo gegen Partei und Führer räsoniert hatten und auf diese Weise unter die Räder der Verfolgungsmaschinerie geraten waren. Verfolgungen, die auf solchen politischen Gelegenheitsäußerungen beruhten, wurden nicht entschä$\operatorname{digt}^{46}$. Aber auch beispielsweise "notorische Rechtsbrecher“, „chronische Alkoholiker und asoziale Psychopathen“ konnten den Gerichten zufolge nicht Träger der geforder-

to $\$ 1(1)$, USEG.

41 OLG Stuttgart, Urteil v. 17.2. 1950, 2 EGR 2, in: RzW 1 (1949/50), S. 248.

42 Otto Küster, Anmerkung zum Urteil der Entschädigungskammer Stuttgart v. 5. 12. 1949, Es 507 (3931), in: RzW 1 (1949/1959), S. 125. Auch bei anderen Gelegenheiten äußerte sich Küster sehr scharf gegen die Entschädigungsansprüche „asozialer Typen“. Vgl. etwa ders., Anmerkung zum Urteil des OLG Stuttgart vom 6. 6. 1950, EGR 16 - Entschädigung, in: RzW 1 (1949/50), S. 340.

43 OLG Stuttgart, Urteil v. 6.6. 1950, EGR 18, in: RzW 1 (1949/50), S. 439. Siehe auch OLG Bremen, Urteil v. 8.7. 1952, WE 16/52, in: RzW 3 (1952), S. 298.

44 Ringelmann in Protokoll der 255. Sitzung des Bundestags-Ausschusses für Rechtswesen und Verfassungsrecht am 7.5. 1953, BA, B141/618.

45 Graf Finckenstein (Bundesinnenministerium), ebenda.

46 OLG Stuttgart, Urteil v. 9.6. 1950, EGR 14, in: RzW 1 (1949/50), S. 315; OLG Stuttgart, Urteil v. 6.6. 1950, 
ten politisch ernsthaften und sittlich gefestigten politischen Überzeugung $\operatorname{sein}^{47}$. Die Schwierigkeit, eine solche nachzuweisen, brachte aber auch etwa Personen um ihre Entschädigung, die während des Krieges Auslandssender gehört hatten und dafür eingesperrt worden waren ${ }^{48}$. Hier genügte ebenfalls nicht der Nachweis allein, daß sie wegen dieses Vergehens verfolgt worden waren, entscheidend war die zugrundeliegende sittlich gefestigte politische Anschauung. Die giftigsten Blüten trieb diese Rechtskonstruktion aber im Falle der irrtümlich Verfolgten. So urteilten die Gerichte wiederholt, daß keinen Anspruch auf Entschädigung habe, „wer für einen Gegner oder Staatsfeind gehalten worden war, ohne es zu sein “49. War also jemand aufgrund einer Namensverwechslung verfolgt worden, so wurde dies als Versehen, aber nicht als entschädigungswürdige Verfolgung betrachtet. Hingegen ging beispielsweise Württemberg-Baden auf Betreiben Küsters einen anderen Weg: hier wurden auch irrtümlich verfolgte Personen entschädigt ${ }^{50}$.

Die Auslegung, daß eine zur Entschädigung berechtigende Verfolgung aus einer Tat resultiert haben mußte, die auf einer sittlich gefestigten politischen Grundeinstellung beruhte, führte aber auch zu anderen "ungeheuren Ergebnissen“. Beispielsweise wurden wegen Rassenschande zu jahrelangem Zuchthaus verurteilte Personen „von der Wiedergutmachung ausgeschlossen ... mit der Begründung, sie hätten den Geschlechtsverkehr nicht aus sittlich gefestigter Auffassung gepflogen "51. So wies etwa die Wiedergutmachungskammer Stuttgart im August 1950 die Forderung eines Klägers nach Entschädigung für seine $\mathrm{Haft}$ wegen Rassenschande $a b$, da es diesem ausschließlich um die "Befriedigung der Geschlechtslust“ gegangen sei ${ }^{52}$. Adolf Arndt, Wiedergutmachungsspezialist der SPD, schlug angesichts dessen erzürnt vor, „Richtern, die derartige Urteile fällten, die Akten um die Ohren zu schlagen“ ${ }^{53}$.

Doch stand Arndt mit seiner Verurteilung solchen Sittenrichtens am Anfang der fünfziger Jahre weitgehend allein. Als beispielsweise die Wiedergutmachungsreferenten der Länder Mitte 1950 die Frage diskutierten, inwiefern Frauen, die wegen sexueller Beziehungen mit Kriegsgefangenen in ein Konzentrationslager gesteckt worden waren, (Auerbach sprach von „erotisch Verfolgten”) Entschädigungsansprüche besäßen, wurde diese Forderung allgemein äußerst skeptisch beurteilt. Küster unterstrich unter dem Beifall der übrigen Anwesenden, daß in Württemberg-Baden nur solche Fälle anerkannt würden, in der derartige Taten in den Rahmen einer

EGR 16, in: RzW 1 (1949/50), S. 339; LG München, Urteil v. 16.11. 1950, EK 160/50, in: RzW 2 (1951), S. 93.

${ }^{47}$ LG Stuttgart, Urteil v. 20.4. 1950, EGR 103, in: RzW 1 (1949/50), S. 315; LG Stuttgart, Urteil v. 25. 4. 1950, EGR 30, in: RzW 2 (1951), S. 93.

48 Adolf Arndt in Protokoll der 255. Sitzung des Bundestags-Ausschusses für Rechtswesen und Verfassungsrecht am 7.5. 1953, BA, B 141/618.

49 LG Stuttgart, Urteil v. 1. 8. 1950, EGR 127, in: RzW 2 (1951), S. 94. Siehe auch OLG Stuttgart, Urteil v. 6.6. 1950, EGR 16, in: RzW 1 (1950), S. 339; LG München, Urteil v. 27.11. 1950, EK 139/50, in: RzW 2 (1951), S. 94 .

$50 \mathrm{Vgl}$. Otto Küster, Anmerkungen zum Urteil des OLG Stuttgart, v. 6.6. 1950, EGR 16 - Entschädigung, in: RzW 1 (1949/50), S. 339 f.

51 Arndt in Protokoll der 252. Sitzung des Bundestags-Ausschusses für Rechtswesen und Verfassungsrecht am 17.4. 1953, BA, B $141 / 618$.

52 Wiedergutmachungskammer Stuttgart, Urteil v. 1.8. 1950, ES 1474 ERG 127, in: RzW 2 (1951), S. 72.

53 Protokoll der 252. Sitzung des Bundestags-Ausschusses für Rechtswesen und Verfassungsrecht am 17.4. 1953, BA, B 141/618. 
politischen Gesamtüberzeugung fielen, denn prinzipiell seien diesbezügliche Verbote auch in anderen Kultur- und Rechtsstaaten üblich gewesen ${ }^{54}$.

Immer wieder stößt man also auf dasselbe Grundproblem: Nicht die Tatsache oder die Schwere der erlittenen Verfolgung bildete die Grundlage der Entschädigungsberechtigung, sondern die Motivation der zugrundeliegenden Verfolgung, sei es auf seiten des Verfolgten oder auf der der Verfolger. Dabei spitzte sich die Frage regelmäßig auf die Definition der spezifisch nationalsozialistischen Verfolgung zu. Diese Unterscheidung spielte insbesondere auch hinsichtlich der Ansprüche der aufgrund des Erbgesundheitsgesetzes Zwangssterilisierten, die bereits bei den Verhandlungen um das Entschädigungsgesetz umstritten gewesen waren, eine herausragende Rolle. Doch überwog bei den zuständigen deutschen Stellen die Meinung, daß diese keine Ansprüche besäßen, da meist keine rassischen, religiösen und politischen Gründe vorlägen ${ }^{55}$. Und Küster betonte: „Entscheidend muß der Grund der Sterilisation sein. Für die normal Sterilisierten (wegen Irrsinn, Erbkrankheiten usw.) soll keine Entschädigung gezahlt werden." 56 Auch hier wurde also in erster Linie auf die Tatsache abgehoben, daß die Zwangssterilisation an sich keine typisch nationalsozialistische Maßnahme und somit das Vorhandensein einer entsprechenden Motivation zur Begründung von Wiedergutmachungsansprüchen erforderlich sei.

Der Zentralverband der Sterilisierten und Gesundheitsgeschädigten kämpfte dagegen Anfang der fünfziger Jahre um die Gleichstellung dieser Gruppe mit den rassisch, religiös und politisch Verfolgten und legte zu diesem Zweck mehreren Länderparlamenten einen Gesetzentwurf vor. Staatssekretär Ringelmann meinte dazu im bayerischen Ministerrat, dies „würde zu einer untragbaren Belastung führen “ ${ }^{57}$. Hieran wird erneut deutlich, wie eng rechtsgrundsätzliche und fiskalische Argumente verwoben waren. In Württemberg-Baden bestimmte Küster im Dezember 1951 per Runderlaß, daß nur derjenige Wiedergutmachung nach dem Entschädigungsgesetz beanspruchen könne, der „aus rassischen und politischen Gründen unfruchtbar gemacht worden ist ... Eugenische Gründe sind in diesem Sinne keine rassischen Gründe “ ${ }^{58}$. Zweck dieses Erlasses war, wie Küster gegenüber dem Rechnungshof seines Landes erläuterte, den Gesetzentwurf des Zentralverbands der Sterilisierten abzufangen ${ }^{59}$.

Aus der Frage nach der Motivation der Verfolger als einer Grundlage des Entschädigungsanspruches resultierte aber auch das gravierende Problem, daß Begründungen aus dem Verfolgungszusammenhang in die Entschädigungsverfahren einflossen. Dieses Problem wurde bereits im Zusammenhang der Ablehnung von Ansprüchen der Nationalverfolgten deutlich, doch spielte es auch etwa im Zusammenhang der Beurteilung von Entschädigungsansprüchen der Zigeuner eine verhängnisvolle Rolle. So teilte Otto Küster bereits Anfang 1950 in einem Runderlaß mit, daß die „Prüfung der Wiedergutmachungsberechtigung der Zigeuner und ,Zigeunermischlinge‘ ... zu dem Ergebnis

54 Protokoll über die Sitzung des Koordinierungsausschusses der 11 Länder, 25. 5. 1950, S. 13, BayMJ, 1101a, H. 4.

55 Siehe dazu etwa Protokoll der Sitzung mit Vertretern des hessischen Innenministeriums, der Fachbehörde Wiesbaden, des Verbands für Freiheit und Menschenwürde und der Gewerkschaft, 5. 12. 1950, HessHStA, Abt. 502, Nr. 2773a.

56 Protokoll über die Sitzung des Koordinierungsausschusses der 11 Länder am 25. 5. 1950, S. 8, BayMJ, 1101a, H. 4.

57 Bayerischer Ministerrat, 22.5. 1951, IfZ-Archiv, NL Hoegner, ED 120, Bd. 367.

58 Staatsanzeiger Württemberg-Baden, Nr. 99, 22. 12. 1951, "Wiedergutmachungsleistungen an Sterilisierte“.

${ }^{59}$ Küster an Rechnungshof Württemberg-Baden, 6. 2. 1952, BadWürtHStA, EA 1/920, Bü. 910. 
geführt“ habe, „daß der genannte Personenkreis überwiegend nicht aus rassischen Gründen, sondern wegen seiner asozialen und kriminellen Haltung verfolgt und inhaftiert worden ist" ${ }^{\text {60 }}$. Philipp Auerbach warf deshalb eingedenk seiner eigenen Erfahrungen als Häftling im Gestapo-Gefängnis Berlin-Alexanderplatz sowie in Auschwitz in einem Rundschreiben Küster rassische Diskriminierung der Zigeuner vor ${ }^{61}$.

Doch auch die Rechtsprechung beteiligte sich seit Beginn der fünfziger Jahre kräftig daran, den Zigeunern die Anerkennung als rassisch Verfolgte streitig zu machen; hierbei handelte es sich um den offenkundigen Fall eines "roll back“ in der Wiedergutmachung. Dies wurde in erster Linie daraus ersichtlich, daß die Gerichte den Zeitpunkt, von dem an eine kollektive Verfolgung der Zigeuner angenommen wurde, immer weiter hinausschoben ${ }^{62}$. Den vorläufigen Schlußpunkt dieser Entwicklung setzte schließlich 1956 der Bundesgerichtshof, der den 1. März 1943, das Datum des sogenannten Auschwitz-Erlasses, in dem die Einlieferung des Großteils der Zigeuner in das Vernichtungslager Auschwitz angeordnet wurde, als Startpunkt ihrer allgemeinen rassischen Verfolgung bestimmte ${ }^{63}$.

Wenn es um die Entschädigungsansprüche dieser Gruppe ging, griffen die Gerichte zudem regelmäßig auf die Argumentation der nationalsozialistischen Verfolger zurück und rechtfertigten frühere Maßnahmen, insbesondere die durch Himmler am 27. April 1940 angeordnete Umsiedlung der Zigeuner nach dem Generalgouvernement, mit militärischen Erfordernissen ${ }^{64}$. Hierbei spielte auch eine Rolle, daß ehemalige nationalsozialistische Rasseforscher wiederholt als Gutachter in einschlägigen Prozessen zu Rate gezogen wurden ${ }^{65}$. Vielfach unterstellte man in Urteilsbegründungen eine zur Erbanlage erklärte Asozialität und Kriminalität der Zigeuner und rechtfertigte auf diese Weise gleichermaßen die Notwendigkeit gegen sie gerichteter polizeipräventiver Maßnahmen in der nationalsozialistischen Zeit wie ihre Entschädigungsunwürdigkeit. So hieß es in einem Urteil des OLG Stuttgart vom 19. Juni 1953: „Vom sicherheitspolizeilichen und militärischen Standpunkt aus gefährlich, weil zu Spionagediensten verlockend, war der den Zigeunern als Rasse eingeborene Wandertrieb. ${ }^{\text {“6 }}$ Hier konnten sich residuale Vorurteile in einer Weise austoben, wie es gegenüber den Juden nicht mehr möglich war: Kein Entschädigungsbeamter oder -richter hätte es gewagt, laut danach zu fragen, ob ein Jude durch sein Verhalten bestimmte gegen ihn gerichtete Maßnahmen herausgefordert habe. Erst Ende 1963 brachte ein Bundesgerichtsurteil schließlich die Wende und erklärte im Gegensatz zur früheren Rechtsprechung und Entschädigungspraxis nunmehr, eine allgemeine rassische Verfolgung der Zigeuner habe bereits seit 1938 bestan$\operatorname{den}^{67}$.

60 Runderlaß E 19 vom 22.2. 1950, zitiert nach J.S. Hohmann, Geschichte der Zigeunerverfolgung in Deutschland, Frankfurt a.M. u. New York 1981, S. 189.

61 Protokoll über die Sitzung des Koordinierungsausschusses der 11 Länder, 17.3. 1950, S. 46, BayMJ, 1091 SA.

62 Wiedergutmachungskammer Karlsruhe, Urteil v. 2.1. 1951, WG II 193, in: RzW 2 (1951), S. 238; OLG Stuttgart, Urteil v. 19.6. 1953, ERG 310, in: RzW 4 (1953), S. 285 f.; OLG München, Urteil v. 19. 5. 1953, WEG 21/53, in: RzW 4 (1953), S.286. Ein abweichendes Urteil fällte hingegen das OLG Frankfurt, das die Anfänge der rassischen Verfolgung der Zigeuner bereits im Jahr 1935 sah. Vgl. OLG Frankfurt, Urteil v. 18.3. 1952, 2 W 722/51, in: RzW 4 (1953), S. 139.

${ }^{63}$ BGH-Urteil v. 7. 1. 1956, IV ZR 211/55 (Koblenz), in: RzW 7 (1956), S. $113 \mathrm{ff}$.

${ }^{64}$ Ebenda; OLG München, Urteil v. 19.5. 1953, W-EG 21/53, in: RzW 4 (1953), S. 286.

65 Vgl. etwa Arnold Spitta, Entschädigung für Zigeuner? Geschichte eines Vorurteils, in: Herbst/Goschler (Hrsg.), Wiedergutmachung, S.398f.

th OLG Stuttgart, Urteil v. 19.6. 1953, RzW 4 (1953), S. 286.

6) BGH-Urteil v. 18. 12. 1963, IV ZR 108/63 (Düsseldorf), RzW 15 (1964), S. 209-211. Zur Vorgeschichte dieser 
Daraus läßt sich das Resümee ziehen, daß unter dem Druck der Finanzierungsprobleme Behörden und Gerichte dazu neigten, den durch das Entschädigungsgesetz der US-Zone vorgegebenen Rahmen weiter zu verengen. Hierbei wirkten sich sowohl der Grundsatz, daß nicht der Schaden an sich, sondern die der Verfolgung zugrundeliegenden Motive der Verfolgten bzw. der Verfolger ausschlaggebend seien, als auch das partielle Fortwirken von Auffassungen und Vorurteilen aus nationalsozialistischer Zeit zuungunsten mancher Verfolgtengruppen aus.

\section{Das Ende der Nachkriegszeit in der Entschädigung?}

a) Der Fall Philipp Auerbach

Zu den Kräften, die auf die Durchführung der Entschädigung Einfluß nahmen, gesellte sich schließlich auch noch die bayerische Landpolizei, indem sie im Januar 1951 das Bayerische Landesentschädigungsamt in München besetzte. Diese Aktion, die der damalige Präsident der Behörde, Philipp Auerbach, zornig als einen „Generalangriff auf die Wiedergutmachung und das Judentum ${ }^{\text {" }} 88$ qualifizierte, hatte erhebliche Folgen: Aus ihr resultierte nicht nur ein längerer Stillstand und eine allgemeine Diskreditierung der Wiedergutmachung, sondern auch ein spektakulärer Prozeß gegen Auerbach und einige andere, an dessen Ende sich dieser das Leben nahm. Den direkten Auslöser für diese Vorgänge lieferte der sogenannte „Fall Wildflecken“, bei dem versucht worden war, im Stile von Gogols „toten Seelen“ für 111 fiktive auswanderungswillige jüdische DP's unter Zuhilfenahme des Bayerischen Landesentschädigungsamtes eine Viertelmillion DM Haftentschädigung von der Stuttgarter Entschädigungsbehörde zu erschwindeln. Doch war dies nur die Spitze des Eisbergs; denn darunter verbargen sich tiefgreifende gesellschaftliche und politische Konflikte um die Stellung der Wiedergutmachung und ihres lautstarken Mentors Philipp Auerbach ${ }^{69}$.

Unter der Leitung Auerbachs hatte sich das bayerische Staatskommissariat für rassisch, religiös und politisch Verfolgte zu einer Einrichtung entwickelt, die eher außerhalb als am Rande der normalen Behördenstruktur stand. Es war eine Art von Superbehörde, die alle Belange der ehemaligen Verfolgten übergreifend regelte und dabei häufig mit den Kompetenzen anderer Stellen zusammenstieß. Justizminister Josef Müller führte deshalb bereits 1948 scharfe Attacken gegen Auerbach und das von ihm geleitete Staatskommissariat und erklärte gegenüber der Presse, dieser führe sich wie ein Gauleiter auf ${ }^{70}$. Und unter vier Augen bedeutete er, Bayern wolle sich nicht von einem jüdischen „König“ regieren lassen ${ }^{71}$. Aber auch im bayerischen Finanzministerium galt das Staatskommissariat als ein Staat im Staate ${ }^{72}$, weshalb dort Ende 1948 die Umbildung in ein Landesamt für Wiedergutmachung beschlossen wurde ${ }^{73}$. Die Hauptveränderung

Wendung in der höchstrichterlichen Rechtsprechung siehe auch Hans Günter Hockerts, Anwälte der Verfolgten. Die United Restitution Organization, in: Herbst/Goschler (Hrsg.), Wiedergutmachung, S. 269-271.

68 Vgl. Süddeutsche Zeitung, 29. 1. 1951, „Entschädigungsamt unter Polizeibewachung“.

69 Vgl. dazu auch Constantin Goschler, Der Fall Philipp Auerbach. Wiedergutmachung in Bayern, in: Herbst/ Goschler (Hrsg.), Wiedergutmachung, S. 77-98.

70 Vgl. etwa Neue Zeitung, 14.10. 1948, "Auerbach heftig angegriffen“.

${ }^{1}$ So berichtet von Hendrik George van Dam. Vgl. Protokoll einer Besprechung van Dams mit Landgerichtsrat Aman, 27. 7. 1951, LG München I, Akten des Prozesses gegen Auerbach und drei Andere, 2 KLs 1/52.

72 Finanzminister Hans Kraus an Ehard, 2. 11. 1948, BayHStA, MA 114240.

73 Verordnung über die Organisation der Wiedergutmachung, 3.1. 1948, in: BayGVOBl, Nr. 23, 19.11. 1948, S. $248 f$. 
bestand darin, daß Auerbach nicht mehr länger eine Doppelstellung als Vertreter des Staates und der Verfolgten einnahm. Man verlieh ihm zwar den wohltönenden Titel "Generalanwalt für Wiedergutmachung", entzog ihm aber zugleich die Verwaltung und Regelung der Wiedergutmachungsansprüche.

Doch ein Jahr später mußte diese Maßnahme weitgehend zurückgenommen werden, da sich die amerikanische Militärregierung an einigen organisatorischen Begleiterscheinungen gestört hatte, die in ihren Augen zu unliebsamen Behinderungen bei der Durchführung des Rückerstattungsgesetzes führten ${ }^{74}$. Deshalb ordnete die bayerische Regierung am 22. November 1949 die Gründung des Landesentschädigungsamtes mit Auerbach als kommissarischem Präsidenten an ${ }^{75}$, womit im Grunde genommen wieder alles beim alten war. Die Strukturelemente einer Sonderverwaltung zur Behebung eines akuten Notstands, die der Situation der unmittelbaren Nachkriegszeit entsprachen und die auch auf den Sonderstatus der Opfer des Nationalsozialismus verwiesen, entwickelten sich aber angesichts der angestrebten "Normalisierung" der gesellschaftlichen Stellung der Verfolgten immer stärker zu einem Anachronismus.

In den Amtsräumen des Landesentschädigungsamtes in der Möhlstraße herrschten unter dem Andrang zahlreicher Verfolgter verschiedenster Nationalitäten chaotische Zustände, die von traditionellen Beamten mit einem gewissen Ekel als „Nachgeburt des Krieges, des Zusammenbruchs, des Chaos " ${ }^{76}$ bezeichnet wurden. Staatssekretär Ringelmann meinte dazu: „Ich wüßte in meiner Verwaltung keinen Mann, dem ich so etwas zumuten könnte. " 77 Die Wiedergutmachung war schon damals kein Feld für ehrgeizige Karrierebeamte. Auerbach hingegen setzte sich hemdsärmelig und häufig unter beträchtlichem Getöse für die Interessen seiner Klientel ein. Seine Devise war, es sei nicht möglich, die Wiedergutmachung im Stile einer normalen Behörde durchzuführen, vielmehr müsse dies nach kaufmännischen Gesichtspunkten geschehen ${ }^{78}$. Er besaß den Ehrgeiz, die Wiedergutmachung soweit als möglich ohne Haushaltsmittel zu finanzieren, und solche Töne vernahm man im bayerischen Finanzministerium durchaus wohlgefällig. Lange Zeit hoffte Auerbach, und mit ihm auch die sparsamen Herren am Odeonsplatz, „daß es möglich sein werde, die Kosten der Wiedergutmachung allein aus dem beschlagnahmten nationalsozialistischen Gut zu decken "79.

Sein Hauptverdienst in den Augen seiner Vorgesetzten war aber, daß er die ihm zu Beginn seiner Amtszeit ausdrücklich gestellte Aufgabe, möglichst bald die DP's aus dem Land zu bringen ${ }^{80}$, mit Bravour erledigte. Der Ministerrat hatte ausdrücklich gebilligt, daß die DP-Haftentschädigungsansprüche unter der Bedingung der Auswanderung vorzugsweise abgefertigt werden sollten. „Der Grund“, so erklärte Ringelmann vor dem Bayerischen Landtag, „lag darin, daß uns damals jeder DP alles in allem 3 bis 400 Mark im Monat für Verpflegung, Unterkunft, Betreuung, Bewachung usw. kostete und daß trotzdem nicht verhindert werden konnte, daß die DP's eine dauernde Gefähr-

74 Rundschreiben von Kraus an Staatskanzlei etc. vom 14.9. 1949, BayHStA, MInn 79667.

75 Zweite Verordnung über die Organisation der Wiedergutmachung vom 22.11. 1949, in: BayGVOBl, Nr. 27, 29.11. 1949, S. 276.

${ }^{76}$ Aussage v. Sebastian Endres, Untersuchungsausschuß zur Prüfung der Vorgänge im Landesentschädigungsamt (UA.LEA), 17. Sitzung, 25.1. 1952, S. 15, BayLt-Archiv.

77 Aussage v. Ringelmann, UA.LEA, 4. Sitzung, 23.8. 1951, S. 37, BayLt-Archiv.

78 Aussage v. Staatsanwalt Hölper, UA.LEA, 2. Sitzung, 16.8. 1951, S.47; Aussage v. Ringelmann, UA.LEA, 4. Sitzung, 23. 8. 1951, S. $36 \mathrm{ff}$. , BayLt-Archiv.

79 Aussage v. Ringelmann, UA.LEA, 4. Sitzung, 23.8. 1951, S. 75, BayLt-Archiv.

${ }^{80}$ Aussage v. Seifried, UA.LEA, 6. Sitzung, 31.8. 1951, S. 106, BayLt-Archiv. 
dung der öffentlichen Sicherheit darstellten." Zwar hatten die deutschen Verfolgten gegen die bevorzugte Bearbeitung der DP-Ansprüche protestiert, doch hielt Ringelmann dagegen, es seien 80.000 Leute gewesen, „die auf diese Weise mit je 500 Mark aus dem Land entfernt werden konnten ${ }^{\text {" }}{ }^{81}$. Entschädigung wurde hier also zu einem Instrument der DP-Auswanderung. Dies tatkräftig durchgeführt zu haben, galt als persönliche Ruhmestat Auerbachs. Bei der Erledigung dieser Aufgabe wurde so manches Auge zugedrückt. In vielen Fällen hatten Bürgermeister und sogar Polizeibehörden, die gleichfalls daran interessiert waren, die DP's so schnell wie möglich loszuwerden, die Abwicklung dadurch beschleunigt, daß sie bereitwillig an Fälschungen der notwendigen Bescheinigungen teilnahmen ${ }^{82}$.

Bei dem Versuch, gleichzeitig die (keineswegs homogenen) Interessen der Verfolgten als auch des bayerischen Staates zu verfechten, geriet Auerbach schließlich zwischen alle Fronten. Zu seinem Erzfeind Josef Müller gesellte sich auch die VVN, nachdem sich Auerbach im Mai 1949 endgültig von ihr getrennt hatte. Auch die Juden in Deutschland standen nur noch partiell hinter ihm: den deutschen Juden war er zu betont jüdisch und den ostjüdischen DP's zu deutsch, zudem irritierte sein Machtbewußtsein. Bedeutender war aber die Gegnerschaft der ausländischen jüdischen Organisationen: für ihren Geschmack vertrat er zu intensiv die Interessen der Juden in Deutschland. Vor allem die Jewish Restitution Successor Organization (JRSO) befand sich in einem Dauerkonflikt mit Auerbach und sann bereits seit längerem darüber nach, ihn loszuwerden ${ }^{83}$. Dabei wurde sie auch von dem stellvertretenden amerikanischen Hohen Kommissar Benjamin B. Buttenwieser unterstützt, der im Zivilleben ein Mann der Wall Street war und zugleich auch dem American Jewish Committee angehörte ${ }^{84}$.

In dem Bestreben, die Entschädigung möglichst schnell abzuwickeln und eine große Anzahl von Anträgen zu erledigen, wurden die bürokratischen Grundsätze im Landesentschädigungsamt häufig ignoriert. Gerüchte, daß dort nicht alles ordnungsgemäß funktionierte, kursierten bereits früh, doch reagierte das Finanzministerium auf die wachsende Flut der Anschuldigungen zunächst zurückhaltend, lagen solche doch schon allein in der Natur des Amtes. Dazu kam, daß angesichts der Nützlichkeit der Tätigkeit Auerbachs nicht immer alle Mißstände auf die Goldwaage gelegt wurden. So waren auch der Staatskanzlei frühzeitig Hinweise zu Auerbachs fragwürdigem Doktortitel, der später entscheidend mit zu seiner Verurteilung beitrug, bekannt, doch hieß es dort: „Aber für die entscheidende Frage, wie man mit diesen unzähligen DP's ... in irgendeiner Form fertig werden kann, ... hat die Frage des ,Doktors' keine so entscheidende Rolle gespielt. ${ }^{\text {8 } 85}$

Schließlich war es der amerikanische Landeskommissar für Bayern, George N. Shuster, der den Stein ins Rollen brachte. In einer dramatischen Sitzung am 26. Januar 1951 eröffnete er Ministerpräsident Ehard, das Münchner Militärdistriktsgericht habe wegen

${ }^{81}$ Bayerischer Landtag, 2. Wp. 1950-1954, 186. Sitzung am 10. März 1954, Stenographische Berichte, S. 930.

${ }^{82}$ Hoegner im bayerischen Ministerrat, 20.2. 1951, IfZ-Archiv, NL Hoegner, ED 120, Bd. 366.

${ }^{83} \mathrm{Vgl}$. auch unten, Abschnitt II. 2.

84 Jacob Blaustein an Harry Greenstein, 3. 9. 1949, YIVO-Archiv, AJC-files, RG 347, GEN-12, Box 22; Paul W. Freedman (American Jewish Committee), „Auerbach Jailed“, 20.3. 1951, S. 17-19; ders., „The Philipp Auerbach Tragedy“, 17.9. 1952, S. 30-33, YIVO-Archiv, AJC-files, FAD 1, RG 347, Box 36. Dort findet sich eine ausgezeichnete Analyse der Hintergründe der Koalition gegen Auerbach und vor allem der jüdischen Opposition.

85 Aussage v. Gumppenberg (Ministerialrat in der bayer. Staatskanzlei), UA.LEA, 18. Sitzung, 1.2. 1951, S. $32 \mathrm{f}$., BayLt-Archiv. 
angeblicher Fälschungen im Bayerischen Landesentschädigungsamt angeordnet, dieses zu schließen und die Akten zu beschlagnahmen ${ }^{86}$. Um die Angelegenheit in deutscher Hand zu behalten, mußte Ehard die deutsche Staatsanwaltschaft einschalten. Noch in derselben Nacht besetzte ein großes Polizeiaufgebot das Landesentschädigungsamt $^{87}$, und am 10. März wurde Auerbach schließlich verhaftet.

Kräftig gefördert durch seinen Intimfeind, Justizminister Müller, ergoß sich nun eine wahre Schlammflut über Auerbach und das Landesentschädigungsamt. Die Arbeit dort wurde für einen längeren Zeitraum völlig blockiert, da die Polizei monatelang Räume und Arbeitskräfte in Beschlag nahm und sämtliche Akten und Anträge kontrollierte. Polizeivizepräsident Weitmann entwickelte in der Angelegenheit eine verhängnisvolle Eigeninitiative, indem er sich quasi zur übergeordneten Entscheidungsinstanz aufwarf, wobei er es insbesondere auf die DP's abgesehen hat$\mathrm{te}^{88}$. Da die Fälschungen hauptsächlich diese Gruppe betrafen, wurde nun die bisherige Situation gerade umgekehrt und ihre Ansprüche extrem langsam bearbeitet ${ }^{89}$. Auch etwa in Hessen wurde wegen dieser Vorgänge die Bearbeitung von DP-Anträgen vorläufig eingestellt ${ }^{90}$. Während die deutsche Öffentlichkeit ihre Vorurteile über die Empfänger der Wiedergutmachung bestätigt sah, erschien diese Aktion vor allem bei den Verfolgten sowie im Ausland als ein schwerer Schlag gegen eine zügige Wiedergutmachung ${ }^{91}$. Gegen den eingetretenen Stillstand der Entschädigung richteten sich zahlreiche Proteste, darunter auch ein „äußerst unangenehmes“ Schreiben McCloys ${ }^{92}$.

Der Prozeß gegen Auerbach und drei weitere Mitangeklagte fand in einer politisch und emotional hochgradig aufgeladenen Atmosphäre statt, stand hier doch der prominenteste Jude Bayerns, Mitglied des Direktoriums des Zentralrates der Juden in Deutschland, gerade sieben Jahre nach Kriegsende einem überwiegend aus ehemaligen Nationalsozialisten zusammengesetzten Gericht gegenüber. Als schließlich am 14. August 1952 das Urteil verkündet wurde, waren etliche zumal der schwersten Vorwürfe fallengelassen worden, doch erkannte das Gericht für passive Bestechung, Untreue, Amtsunterschlagung, versuchten Meineid und unbefugte Führung eines Doktortitels auf zwei Jahre und sechs Monate Gefängnis sowie 2.700 DM Geldstrafe $^{93}$. Mit Ausnahme der Vorwürfe wegen seines zu Unrecht geführten

${ }^{86}$ Aussage v. Hirsch, UA.LEA, 15. Sitzung, 7. 12. 1951, S. 32 f., BayLt-Archiv.

87 Aussage v. Ehard, UA.LEA, 10. Sitzung, 12.10. 1951, S.59ff., BayLt-Archiv; Bericht von Justizminister Müller über die Besetzung des Landesentschädigungsamtes, Bayerischer Landtag, 2. Wp. 1950-1954, 11. Sitzung am 8. 2. 1951, Stenographische Berichte, S. $158 \mathrm{f}$.

88 Siehe dazu etwa den Protest von Finanzminister Zorn, ders. an Weitmann, 19.3. 1951, sowie Verband der Jüdischen Invaliden an die Redaktion des Münchner Merkur, 22.3. 1951, BayHStA, MA 114264.

89 Josef Müller, Protokoll über Besprechung im Justizministerium zwischen Vertretern des Justizministeriums, der deutschen und der amerikanischen Justiz sowie der Polizei am 29.1. 1951, LG München I, Akten des Prozesses gegen Auerbach und drei Andere, 2 KLs 1/52; Friedrich Zietsch im bayerischen Ministerrat, 14. 8. 1951, IfZ-Archiv, NL Hoegner ED 120, Bd. 369.

90 Daniels (HICOG/Property Division) an Alex F. Kiefer (State Department/Property Division), 24. 3. 1951, USNA, RG 59, 262.0041/3-2451.

91 Siehe etwa Max Bachmann (deutscher Generalkonsul in London) an von Trützschler (Auswärtiges Amt), 14. 10. 1952, PA/AA, II 244-13 II, Bd. 9.

92 McCloy an Ehard, 16.5. 1951, BayHStA, MA 114240; Ehard im bayerischen Ministerrat, 22. 5. 1951, IfZArchiv, NL Hoegner, ED 120, Bd. 367.

93 Urteil in der Strafsache gegen Auerbach und drei Andere, S. 2 f., LG München I, 2 KLs 1/52. 
Doktortitels stritt Auerbach jedoch weiterhin alle Vorwürfe $\mathrm{ab}^{94}$. Als gesundheitlich, finanziell und moralisch zerstörter Mann nahm er sich drei Tage nach dem Urteil das Leben.

Strafrechtliche und politische Aspekte sollten bei einer Bewertung sorgfältig auseinanderhalten werden. Juristisch gesehen besteht kein Zweifel, daß Auerbach kein Unschuldslamm war; das gestanden selbst seine Freunde und Gönner ein. Daß es bei der Erfüllung der schwierigen Aufgabe der Fürsorge und Entschädigung der Verfolgten nicht immer vorschriftsmäßig zuging, blieb nicht verborgen, wurde aber lange Zeit nicht als ausschlaggebend bzw. sogar als vorteilhaft für den bayerischen Staat angesehen. Zunächst stand im Vordergrund, daß Auerbach die Vertretung der Interessen der Verfolgten durchaus mit der Wahrung der Interessen der bayerischen Regierung zu verbinden gewußt hatte, was besonders in der Frage der Finanzierung und vor allem der forcierten Auswanderung der DP's deutlich geworden war. Ein vom Bayerischen Landtag eingesetzter Untersuchungsausschuß, der über die Frage, ob Auerbachs vorgesetzte Stellen ihre Dienstaufsicht verletzt hatten (und nicht über die juristische Schuld Auerbachs ${ }^{95}$ ) befinden sollte, erklärte dazu in seinem Abschlußbericht: „Solche Aufgaben waren nicht mit normalen Mitteln und auch nicht von Persönlichkeiten zu lösen, die zwar getreu dem Gesetz arbeiteten, der außergewöhnlichen Lage gegenüber jedoch ziemlich hilflos gewesen wären. ${ }^{\text {“96 }}$ So hatte der jüdische Rechtsanwalt Benno Ostertag während des Prozesses geschrieben: „Auerbach war der Zauberbesen seiner Regierung, den man in die Ecke stellen konnte, wann immer es seiner Regierung lieb war und der kehren mußte, wann immer sie das wollte." 97

Die Affäre Auerbach läutete das Ende der Nachkriegszeit in der Wiedergutmachung ein. Der bürokratische Sonderstatus dieser Materie, der immer auch eine Sonderstellung der Verfolgten ausdrückte, wurde nun in Bayern endgültig beseitigt. Dies bedeutete auch eine Zäsur für die Arbeit der Entschädigungsbehörden. Karl Heßdörfer sprach von der Auerbach-Affäre als einem Schock, der eine „Art Kontrollzwang“ ausgelöst habe ${ }^{98}$. Die Grundlinien der Entschädigungspraxis wurden künftig nicht mehr in der Möhlstraße, sondern am Odeonsplatz festgelegt: Nun setzte die Herrschaft des Finanzministeriums ein, die bis heute anhält.

94 "Politisches Testament" Auerbachs, 14.8. 1952, LG München I, Akten des Prozesses gegen Auerbach und drei Andere, 2 KLs 1/52.

95 Juliane Wetzel schreibt irrtümlicherweise, der Untersuchungsausschuß des Bayerischen Landtags habe Auerbach von den in seinem Prozeß gegen ihn erhobenen Vorwürfen freigesprochen, womit sie eine Fehleinschätzung des von Bruno Weil geleiteten "Committee for Fair Play for Auerbach“ übernimmt. Vgl. dies., Jüdisches Leben in München 1945-1951. Durchgangsstation oder Wiederaufbau?, München 1987, S. 62. Richtig daran ist nur, daß dieses Gremium, das keine forensischen Funktionen ausübte, die besonderen Umstände des Verhaltens Auerbachs stärker unter politischen Gesichtspunkten würdigte und ihm von daher eher gerecht wurde.

96 Schlußbericht des Untersuchungsausschusses zur Prüfung der Vorgänge im Landesentschädigungsamt (Anm. 4), S. 15.

97 Benno Ostertag, "Auerbach und die bayerische Regierung“, in: Allgemeine Wochenzeitung der Juden in Deutschland, 22.2. 1952.

98 Karl Heßdörfer, Die Entschädigungspraxis im Spannungsfeld von Gesetz, Justiz und NS-Opfern, in: Herbst/ Goschler (Hrsg.), Wiedergutmachung, S. 233. 


\section{b) Der Fall Otto Küster}

Eine ähnliche Entwicklung zeigte sich auch außerhalb Bayerns. Otto Küster hatte als Staatsbeauftragter für Wiedergutmachung in Württemberg-Baden seinem Land den Ruf als „Musterländle“ der Wiedergutmachung erworben. Doch machte er es dabei offenbar nicht allen recht. Das Finanzministerium störte sich daran, daß Küster das Entschädigungsgesetz durch Erlasse und Verordnungen häufig so weit wie möglich zugunsten der ehemaligen Verfolgten interpretierte. Daß er dabei nicht wahllos vorging, offenbarte die Untersuchung der Durchführung des Entschädigungsgesetzes ${ }^{99}$. Ein einflußreicher Widersacher erwuchs Küster aber auch in Gebhard Müller, der nach der Vereinigung des Südweststaates 1952 der erste Ministerpräsident Baden-Württembergs wurde. Nach dem Globalabkommen mit Israel, an dessen Zustandekommen der württembergbadische Wiedergutmachungsbeauftragte als stellvertretender Delegationsleiter beteiligt war, hatte Müller Küster zufolge in internen CDU-Zirkeln kolportiert, dieser sei „Adenauer und Schäffer in den Rücken gefallen, hätte die Bundesrepublik an die Juden verraten und uns um viele Milliarden gebracht;“ zudem verschwende er „die Steuergelder Württemberg-Badens an die Juden. Wenn er in Stuttgart an die Macht komme, werde er aufräumen “ ${ }^{100}$. Nachdem Müller Ministerpräsident geworden war, drehte sich in der Tat alsbald der Wind: Aus der Regierungserklärung wurde der ursprünglich vorgesehene Satz, die Regierung werde „die Wiedergutmachungsarbeit mit dem bisherigen Ernst weiterführen“, gestrichen. Der neue Justizminister, Wolfgang Haussmann, teilte Küster überdies mit, zu den Richtlinien der Politik des Herrn Ministerpräsidenten gehöre es, „daß sich das Land Baden-Württemberg in der Wiedergutmachung nicht mehr so vordränge wie bisher" ${ }^{101}$.

Schon früher hatte der württemberg-badische Ministerrat den Auerbach-Skandal zum Anlaß genommen, die heimische Entschädigungspraxis einer Sonderuntersuchung durch den Rechnungshof zu unterziehen. Als der Bericht nach längerer Zeit vorlag, lieferte er dem Finanzministerium die gewünschte Munition für die Forderung, daß grundlegende Erlasse des Justizministeriums in Wiedergutmachungssachen künftig an seine interne Zustimmung gebunden sein sollten ${ }^{102}$. Ein Vertreter des Landesfiskus hielt Küster vor, die Beanstandungen des Rechnungshofes zeigten, „daß seitens der Wiedergutmachungsbehörden Entscheidungen getroffen worden sind, die vom Standpunkt des Steuerzahlers nicht gebilligt werden können“. Das Finanzministerium gründete darauf die Forderung, daß ihm künftig „der erforderliche Einfluß“ gewährt werden müsse. Gegen den Vorwurf, daß die Entschädigungsbehörden „die fiskalischen Interessen außer Acht gelassen hätten“, verteidigte sich jedoch Küster, Baden-Württemberg hätte „weit weniger Aufwendungen gehabt als andere Länder, die nicht im Rufe der Wiedergutmachungsfreundlichkeit stehen. $85-90 \%$ der beantragten Renten wurden abgewie-

99 Christian Pross neigt dagegen in seiner Darstellung zu einer gewissen Glorifizierung Küsters, der ihm hauptsächlich als der "gute“ Widerpart zum „bösen“ Bundesfinanzministerium dient. Diese dichotomische Betrachtungsweise, die die Geschichte der Wiedergutmachung als Western beschreibt, ist zwar sehr griffig, wird aber der Komplexität der Küsterschen Position nicht gerecht. Vgl. ders., Wiedergutmachung. Der Kleinkrieg gegen die Opfer, Frankfurt a.M. 1988, S. 78-91.

100 Otto Küster an Franz Böhm, 11. 7. 1954, abgedruckt in: Freiburger Rundbrief, September 1954, Nr. 25/28, S. 8.

101 Viktor Renner, Landtag von Baden-Württemberg, 1. Wp. 1952-1956, 43. Sitzung am 5. 8. 1954, Stenographische Berichte, S. 1809.

102 Erklärung Küsters gegenüber den Mitarbeitern des Amts für Wiedergutmachung, Stuttgart, 1.7. 1954, in: Freiburger Rundbrief, September 1954, Nr. 25/28, S. 5. 
sen, das“, so Küster, „ist ihnen in den anderen Ländern nicht gelungen“ ${ }^{103}$. Dieser eigentümliche Stolz illustriert erneut, daß Küsters Ruf als einer der Heroen der Wiedergutmachung nicht auf verschwenderischer Großzügigkeit beruhte.

Schließlich wurde aufgrund einiger dubioser Anschuldigungen gegen Küster seitens eines entlassenen Mitarbeiters ein Untersuchungsausschuß eingesetzt. Unter den gesammelten Vorwürfen rangierte auch die Dienstbeschwerde von Bundesfinanzminister Fritz Schäffer vom 26. Februar 1954, der auf eine Philippika Küsters gegen die Mängel des unter der Regie seines Ministeriums entstandenen Bundesergänzungsgesetzes hin seinen Mitarbeitern den dienstlichen Umgang mit Küster verboten hatte ${ }^{104}$. Küster hatte in einem Vortrag Ende 1953 in Freiburg „in gewissem Sinn zum Ungehorsam aus höherem Gehorsam" gegenüber dem Geist der Wiedergutmachung aufgefordert ${ }^{105}$. Die Untersuchung durch Landgerichtspräsident Teufel, „einem“, wie Küster es formulierte, „würdigen Reaktionär aus der idyllischen Fastnachts-Stadt Rottweil “106, erbrachte schließlich zwar ein 50-seitiges Dossier, aber keinerlei haltbare Vorwürfe.

Doch am 28. Juni 1954 beschloß der Ministerrat, daß künftig alle Anordnungen des Justizministeriums, die von finanzieller Bedeutung für die Wiedergutmachung waren, der Zustimmung des Finanzministeriums bedurften ${ }^{107}$. Dies war der entscheidende Schlag gegen die Unabhängigkeit der Entschädigungspraxis. Zugleich versuchte man auch die persönliche Unabhängigkeit Küsters zu beschneiden. Er wurde aufgefordert, sich verbeamten zu lassen, was Ministerpräsident Müller ihm gegenüber damit schmackhaft gemacht hatte, „sonst hätte man ja nicht einmal die Möglichkeit eines Disziplinarverfahrens gegen ihn ${ }^{* 108}$. Die angebotene Beamtenstelle, die eine deutliche Herabstufung seiner Position bedeutet hätte, war für Küster unannehmbar, und genau darauf zielte dieses Manöver auch. So wurde ihm am 30. Juni mit Wirkung zum Ende des Jahres gekündigt.

Diese Vorgänge waren ziemlich durchsichtig, weshalb die baden-württembergische Regierung in der Öffentlichkeit heftig kritisiert wurde. In dieser Situation kam ihr zu Hilfe, daß Müller durch die Indiskretion eines Spiegel-Journalisten ein persönlicher Brief Küsters an Franz Böhm, in dem er seinem Zorn über diese Winkelzüge Luft machte, zugespielt wurde. Unter anderem empörte sich Küster dort: „Was die Wiedergutmachung und mich anbelangt, so haben wir keinen Ministerrat, sondern eine Waschküche von tuschelnden Weibern. " 109 Dieser Brief lieferte Müller nun einen Vorwand, Küster schließlich am 4. August fristlos zu entlassen, was bemerkenswerterweise quer durch alle Parteien gebilligt wurde. Nur sein ehemaliger Vorgesetzter, Ex-Justizminister Victor Renner, trat vor dem Stuttgarter Landtag für Küster ein ${ }^{110}$.

103 Niederschrift der Sitzung einer Kommission im baden-württembergischen Staatsministerium zur Prüfung der Wiedergutmachungszahlungen am 11.2. 1954, BadWürtHStA, EA 1/920, Bü.710.

$104 \mathrm{Vgl}$. Otto Küster und die individuelle Wiedergutmachung, in: Freiburger Rundbrief, September 1954, Nr. 25/28, S.6; Anlage 1 zu den Verhandlungen des Landtags von Baden-Württemberg, 1. Wp. 1952-1956, 43. Sitzung am 5.8. 1954: Bericht des Justizministeriums über die Beziehungen zwischen Rechtsanwalt Küster und dem Lande Baden-Württemberg, Stenographische Berichte, S. 1838.

105 Otto Küster, Das Gesetz der unsicheren Hand. Vortrag in Freiburg am 3. 12. 1953, in: Freiburger Rundbrief, Februar 1954, Nr. 21/24, S. 9.

106 Küster an Böhm, 11.7. 1954, in: Freiburger Rundbrief, September 1954, Nr. 25/28, S. 8.

107 Viktor Renner, Klarheit und Wahrheit, ebenda, S. 20.

108 Ebenda.

109 Küster an Böhm, 11.7. 1954, ebenda, S. 8.

${ }^{110}$ Landtag von Baden-Württemberg, 1. Wp. 1952-1956, 43. Sitzung am 5.8. 1954, Stenographische Berichte, S. 1807-1823. 
Ein zeitgenössischer Kommentar bemerkte, der „Fall Küster“ sei letztlich „aus dem unmöglichen Bestreben entstanden, die Wiedergutmachung in den normalen Ablauf des sich konsolidierenden öffentlichen Lebens einzubauen. Man wollte ihr Ärgernis beseitigen." 111 Mit dieser Erklärung ist auch die Gemeinsamkeit der Fälle Auerbach und Küster auf den Punkt gebracht. In Bayern wie in Baden-Württemberg kollidierte die Sonderstellung der Wiedergutmachung im Behördenapparat mit einer politischen Konjunktur, die auf eine „Normalisierung“ gegenüber den Verfolgten des Nationalsozialismus drängte. Auerbach und Küster waren persönlich höchst verschieden: der eine ein hanseatisch-jüdischer Chaot, der andere ein schwäbisch-protestantischer Pedant; der eine kein Beamter, weil die Regierung seinen Bereich nicht allzu fest etablieren mochte, der andere, weil es seinen persönlichen Prinzipien widerstrebte. Doch erlitten sie das gemeinsame Schicksal, als bürokratische Außenseiter, die ihre außergewöhnliche Aufgabe mit ungewöhnlichen Methoden verfolgten, verstoßen zu werden.

Auch bei den Auswirkungen auf die Entschädigungspraxis ergeben sich Parallelen. Das amtlich inszenierte Kesseltreiben gegen Küster führte in Baden-Württemberg gleichfalls zu einer tiefen Verunsicherung der Entschädigungsbehörden. Schon Anfang 1954 hatte dieser geklagt, daß die verängstigten Landesämter für Entschädigung kaum noch Leistungen bewirkten, da ihnen die Angst vor dem Rechnungshof im Nacken sitze $^{112}$. Dieser atmosphärische Druck oder Kontrollzwang, wie es Heßdörfer im Zusammenhang des bayerischen Beispiels nannte, wurde dadurch, daß die Durchführung der Entschädigung auch hier weitgehend unter die Herrschaft des Fiskus fiel, keineswegs gemindert. In den Finanzministerien, Rechnungshöfen und ihren Behörden wurden und werden bis auf den heutigen Tag Wiedergutmachungsleistungen in erster Linie unter dem Aspekt der Belastung des Staatshaushalts und des Steuerzahlers betrachtet.

Franz Böhm schrieb deshalb unter dem Eindruck der Entlassung Küsters: „Moralität, Gewissen, Wiederherstellung einer zerstörten und beleidigten Ordnung, Rechtschaffenheitsansehen, ethischer Kredit - das sind alles Aktivposten, die sich in den Haushaltsvoranschlag des Bundes und der Länder nicht mit Zahlen einsetzen und nicht in Geld abschätzen lassen. Die Lücke, die Schadensersatzzahlungen in den Etat reißen, ist das einzig Reale, das sich auf Heller und Pfennig nachweisen läßt. Deshalb haben auch die Finanzminister bei keiner anderen Ausgabenposition so viel Mitleid mit dem Steuerzahler wie bei dieser. ${ }^{113}$ Dies war nun in gewissem Sinne auch der Preis dafür, daß die Entschädigung zu einer Aufgabe der öffentlichen Hand und damit verstärkt zum Gegenstand innenpolitischer Konkurrenzzwänge geworden war.

111 Vgl. Wort und Wahrheit, „Rückspiegel“, September 1954, zit. nach Freiburger Rundbrief, September 1954, Nr. 25/28, S. 17

112 Küster an Staatsministerium, 23. 2. 1954 sowie Rechnungshof Baden-Württemberg an baden-württembergisches Finanzministerium, 9.4. 1954, BadWürtHStA, EA 1/920, Bü. 710.

113 Franz Böhm, Zum Ausscheiden Otto Küsters, in: Freiburger Rundbrief, September 1954, Nr. 25/28, S. 13. 


\section{Rückerstattung unter amerikanischer Aufsicht}

\section{OMGUS und HICOG als Motoren der Rückerstattung}

Anders als bei dem unter deutscher Regie durchgeführten Entschädigungsgesetz der US-Zone blieb die amerikanische Militärregierung bzw. Hohe Kommission im Falle des Militärregierungsgesetzes Nr.59, das die Rückerstattung wiederauffindbaren Eigentums regelte, auch an der Durchführung stark interessiert. Nicht umsonst war dieses Gesetz durch die Militärregierung erlassen worden: Die deutsche Seite war nicht bereit gewesen, die politische Verantwortung für ein Gesetz, das so stark von den amerikanischen und jüdischen Interessen geprägt war, zu übernehmen ${ }^{114}$. Die erheblichen Gegensätze, die bei der Entstehung des Gesetzes deutlich wurden, kamen natürlich erst recht bei der Durchführung zum Tragen, zumal diese überwiegend in die Zeit nach Gründung der Bundesrepublik fiel, als wachsende Souveränitätsansprüche immer stärker mit dem besatzungsrechtlichen Grundcharakter eines solchen Militärregierungsgesetzes kollidierten.

Zweck des Gesetzes war die Regelung der Rückerstattung wiederauffindbaren Vermögens, das in der Zeit vom 30. Januar 1933 bis 8. Mai 1945 aus Gründen der Rasse, Religion, Nationalität, Weltanschauung oder der politischen Gegnerschaft zum Nationalsozialismus ungerechtfertigt entzogen worden war, an die rechtmäßigen Eigentümer. Es forderte zudem die „schleunige Rückerstattung im größtmöglichen Umfang “115. Dieses Ziel des Rückerstattungsgesetzes wurde dem amerikanischen Hohen Kommissar auch noch einmal eigens zur Pflicht gemacht: Es gehörte zu den Aufgaben, die er bis zur Ablösung des alliierten Besatzungsstatuts erledigen sollte ${ }^{116}$. Das Amt des amerikanischen Hohen Kommissars konkretisierte dies und nannte den 31. Dezember 1951 als vorgesehenen Endpunkt für die Durchführung des Gesetzes ${ }^{117}$. Eine solch ehrgeizige Absicht lag natürlich im Interesse der Geschädigten. Zugleich ging es aber vor allem darum, so bald als möglich Ruhe auf diesem konfliktträchtigen Gebiet einkehren zu lassen. Daneben wies Washington am 17. November 1949 McCloy u.a. auch an, eine Übereinkunft mit seinen britischen und französischen Kollegen zu suchen, um die deutsche Regierung gemeinsam zum baldigen Erlaß eines möglichst eng an das amerikanische Militärregierungsgesetz Nr. 59 angelehnten bundeseinheitlichen Rückerstattungsgesetzes zu bewegen ${ }^{118}$. Ziel der amerikanischen Politik war also zunächst einmal schleunige Abwicklung der Rückerstattung in der US-Zone und dann nach Möglichkeit Ausdehnung der amerikanischen Regelung auf die Bundesrepublik ${ }^{119}$.

Aber bereits im Sommer 1950 wurden die Harmonisierungsbestrebungen zugunsten der beschleunigten Durchführung des Militärregierungsgesetzes Nr. 59 in der US-Zone

114 Vgl. Drittes Kapitel, Abschnitt II. 4.

$115 \int 1$, Gesetz Nr. 59 der amerikanischen Militärregierung (USREG), in: Amtsblatt der amerikanischen Militärregierung Deutschlands, Ausgabe G, 10.11. 1947, S.1.

116 Siehe u.a. C.A. McLain (HICOG/General Counsel) an Executive Secretary, o.Dat. (ca. Nov./Dez.1949), IfZ-Archiv, MF 260, OMGUS-LD 17/200-2/8.

117 Siehe u. a. McCloy an Ehard, 23. 1. 1950, BayMJ, 1101a, H. 7.

118 \34 der Policy Directive vom 17. 11. 1949. Siehe Daniels an State Department, 29.6. 1950, USNA, RG 59, 262.0041/6-2950.

119 Siehe auch einen Bericht von Joel D. Wolfsohn (AJDC) über eine Besprechung im State Department am 2.8. 1949, in der Übereinstimmung mit der entsprechenen Auffassung der amerikanischen jüdischen Organisationen geäußert wurde: Wolfsohn an Simon Segal, 3.8. 1949, YIVO-Archiv, RG 347, AJC, Records GEN-10, Box 276. 
aufgegeben. Grund dafür waren die wachsenden Schwierigkeiten bei dem amerikanischen Versuch, "gleichzeitig auf zwei Pferden zu reiten“ ${ }^{120}$, d. h. eine schnelle Abwicklung und eine weitgehende Übernahme der US-Regelung durch Briten und Franzosen zu erreichen. So bat die amerikanische Hohe Kommission Ende Juni das State Department um eine Änderung der bisherigen Richtlinien, die die Harmonisierung der Rückerstattungsgesetzgebung der Bundesrepublik bzw. Bemühungen um den Erlaß eines einheitlichen bundesdeutschen Rückerstattungsgesetzes vorsahen. Weitere Verhandlungen mit den Alliierten erschienen nun als aussichtslos, nachdem mit der Verkündung eines Rückerstattungsgesetzes in der britischen Zone und der geplanten Bestimmung einer Nachfolgeorganisation für erbenloses jüdisches Eigentum in der französischen Zone wenigstens ein Minimum an Einheitlichkeit erreicht worden war ${ }^{121}$. Dazu trat das schwerwiegende Argument, daß die amerikanischen Bestrebungen auf Vereinheitlichung oder Harmonisierung der Rückerstattung deutsche Hoffnungen auf eine Milderung des Gesetzes nährten ${ }^{122}$. Am 19. Juli stimmte US-Außenminister Dean Acheson dem vorgeschlagenen Kurswechsel förmlich zu. Zugleich unterstrich er aber, daß „die Rückerstattungspolitik des Ministeriums hinsichtlich der Auffassung, daß das Programm beschleunigt und so schnell als möglich zum Abschluß gebracht werden soll, unverändert bleibt" ${ }^{\prime 123}$.

Doch bildete der deutsche Widerstand gegen die alliierten Rückerstattungsgesetze, der sich besonders an der als drakonisch verschrienen Regelung in der US-Zone entzündete, ein ernstes Hindernis für dieses Vorhaben. Vor allem seit Gründung der Bundesrepublik forderten zahlreiche Initiativen auf parlamentarischer Ebene, seitens der Verbände der Rückerstattungsgeschädigten sowie auch der Industrie lautstark die Abmilderung der alliierten Rückerstattungsbestimmungen. Als bevorzugter Weg erschien dazu für eine gewisse Zeit die Vereinheitlichung der alliierten Rückerstattungsgesetze - wobei unterstellt wurde, daß dies zu einer gegenüber dem Gesetz der amerikanischen Zone günstigeren Lösung führen würde ${ }^{124}$. Im Zentrum der Agitation gegen die Rückerstattung standen Interessenverbände der Restitutionsgeschädigten. In der US-Zone bildete sich die „Interessensgemeinschaft der Rückerstattungspflichtigen“, die sich im Frühjahr 1950 mit ähnlichen Vereinigungen der anderen Zonen zur „Bundesvereinigung für loyale Rückerstattung " zusammenschloß ${ }^{125}$. Diese Vereinigungen entfalteten eine wirksame lobbyistische Tätigkeit in den Landtagen und im Bundestag. Sie stellten ihre Forderungen unter die Devise einer "loyalen Restitution“, ein Begriff der bewußt mehrdeutig gehalten war. Kritisiert wurden sowohl unbezweifelbare Härten der Rückerstattung, etwa im Falle, daß arisiertes Vermögen unwissend aus zweiter Hand erworben war oder daß kleine Bauern aus Staatshand arisiertes Land gekauft hatten, aber das Spektrum reichte auch bis hin zu einer Ablehnung der Rückerstattung in toto ${ }^{126}$. Die Kritik richtete sich dabei hauptsächlich gegen die Fälle, in denen es sich um jüdische Rückerstattungsforderungen handelte, wobei immer wieder deutlich wur-

\footnotetext{
120 John Rintels (HICOG/Office of General Counsel/Administration of Justice Division), Minutes of Meeting with the High Commissioner, 10.4. 1950, WNRC, RG 466, McCloy Papers, Box 12.

121 Vgl. Fünftes Kapitel, Abschnitt V.1.

122 Daniels an State Department, 29.6. 1950 (Anm. 118).

123 Acheson an HICOG Frankfurt, Property Division, 19. 7. 1950, USNA, RG 59, 262.0041/6-2950.

124 Vgl. dazu auch Schwarz, Rückerstattung nach den Gesetzen der Alliierten Mächte, München 1974, S. 69-95.

125 Highlights of Property Division Activities for May 1950, USNA, RG 59, 262.0041/6-1350.

126 Ebenda. Vgl. auch Schwarz, Rückerstattung nach den Gesetzen der Alliierten Mächte, S. $71 \mathrm{ff}$.
} 
de, wie mäßig das Bewußtsein für die kollektive Verfolgungssituation der Juden unter dem NS-Regime hier entwickelt war. So argumentierte die in Berlin erscheinende Zeitschrift Der Grundbesitz - Untertitel: „Eine Zeitschrift für Eigentümer und Verwalter“ -, daß „ganz allgemein in der Arisierung die tatsächliche Wiedergutmachung gesehen wurde“, denn jüdische Spekulanten hätten in der Inflationszeit in großem Umfang Immobilien zu Spottpreisen errafft ${ }^{127}$.

Einen Zankapfel bildete aber auch das Verfahren der Rückerstattung. Hauptstreitpunkt war dabei der amerikanische Board of Review in Nürnberg, der als oberste Nachprüfungsinstanz alle Urteile der deutschen Rückerstattungsgerichtsbarkeit überwachte und kassieren konnte ${ }^{128} .1950$ wurde daraus der Court of Restitution Appeals (CORA). Neben der zentralen Anmeldestelle in Bad Nauheim war dies die einzige Einrichtung, die direkt unter amerikanischer Kontrolle stand, alle übrigen 47 in der USZone mit der Durchführung der Rückerstattung befaßten Ämter und Gerichte waren deutsch ${ }^{129}$. Daß es kein vergleichbares Kontrollinstrument gab, das die Entschädigungspraxis der deutschen Ämter und Gerichte hätte überprüfen können, verdeutlicht noch einmal das unterschiedliche amerikanische Engagement im Bereich von Rückerstattung und Entschädigung ${ }^{130}$.

Dabei gab es gute Gründe für eine solche Überwachungsinstanz im Bereich der Rückerstattung: Erstens war bei der Auseinandersetzung um die Entstehung des Militärregierungsgesetzes Nr. 59 deutlich geworden, daß es im Widerspruch zu einer Anzahl von herkömmlichen deutschen Rechtsauffassungen stand, insbesondere was den im Bürgerlichen Gesetzbuch verankerten Schutz des gutgläubigen Erwerbers betraf. Hinzu kam, daß beide Seiten, Kläger wie Pflichtige, versuchten, durch Einwirkung auf die Auswahl der Richter für das Rückerstattungsverfahren die Tendenz der Rechtsprechung zu beeinflussen. Bereits im Frühjahr 1948 forderte die amerikanische Militärregierung das American Jewish Distribution Committee (AJDC) ultimativ auf, ihren Vertreter Werner Peiser aus Deutschland zurückzuziehen, nachdem sie erfahren hatte, daß er in den Ländern der amerikanischen Zone die Auswahl der Richter für die Wiedergutmachungskammern der Landgerichte beeinflußt hatte ${ }^{131}$. Umgekehrt wurde Anfang 1949 bekannt, daß besonders in Bayern Bemühungen von interessierter Seite erfolgreich waren, solche Richter zu plazieren, von denen man antijüdische Urteile erwarten konnte ${ }^{132}$. Die Forderung nach der vorzugsweisen Beschäftigung von nichtnationalsozialistisch vorbelasteten Richtern auf diesem Gebiet bereitete allerdings erhebliche praktische Schwierigkeiten: Es gab einfach zu wenige, die dieses Kriterium erfüllten. Hinzu kam, daß die kleine Schar der unbelasteten Richter bereits im Zuge der

127 Der Grundbesitz, Nr. 5, Mai 1949, S. 29.

128 Ausführungs-Verordnung Nr. 4 zum Gesetz Nr. 59 der amerikanischen Militärregierung, in: Amtsblatt der Militärregierung Deutschland, Amerikanisches Kontrollgebiet, Ausgabe K, 1.9. 1948, S.1. Vgl. auch Schwarz, Rückerstattung nach den Gesetzen der Alliierten Mächte, S. $275 \mathrm{f}$.

129 Auflistung der Wiedergutmachungsämter in E.N. Reinsel an den Leiter des Zentralanmeldeamtes in Bad Nauheim, 21.2. 1948, HessHStA, Abt. 502, Nr.2773a.

130 Eine solche Einrichtung schlug noch Ende 1951 der amerikanische Kongreß-Abgeordnete Jacob Javits den US-HICOG-Vertretern vor, doch wurde er belehrt, daß der Zug für eine derartige Maßnahme längst abgefahren sei. Siehe Transcript of Briefing for the Special Sub-Committee of the House Foreign Affairs Committee (German Study Mission), 12.11. 1951 (Anm.6).

131 Charles LaFollette (OMGB) an General George P. Hays, 5. 3. 1948. Sowie Hays an Clay, 18. 3. 1948, IfZArchiv, MF 260, OMGUS, AG 1948/184/1.

132 John M. Raymond an Administration of Justice Branch, Legal Division, Nürnberg, 8. 1. 1949, IfZ-Archiv, MF 260, OMGUS-LD 17/214-2/8. 
Entnazifizierung verschlissen worden war ${ }^{133}$. So war die amerikanische Militärregierung letztlich zu dem Schluß gekommen, besser nicht direkt in die Personalauswahl einzugreifen, sondern die Entscheidungen dieser Richter scharf zu beobachten ${ }^{134}$.

Der hartnäckige deutsche Widerstand gegen eine Rückerstattung nach den Normen des amerikanischen Militärregierungsgesetzes Nr. 59 stellte jedoch die amerikanischen Pläne über einen schleunigen Abschluß des Verfahrens ernstlich in Frage. Das ehrgeizige Ziel der amerikanischen Hohen Kommission, die Abwicklung des Rückerstattungsgesetzes bis Ende 1951 abzuschließen, ging von der Erwartung einer hohen Quote außergerichtlicher Vergleiche aus ${ }^{135}$. Aufgrund kursierender Gerüchte über bevorstehende Milderungen der Bestimmungen sank jedoch auf Seiten der Pflichtigen die Bereitschaft zu derartigen Vergleichen, da sie vielfach, in der Hoffnung, daß sich die rechtliche Lage in der Zwischenzeit zu ihren Gunsten ändern würde, lieber auf dem Weg durch alle Instanzen das Verfahren in die Länge zogen ${ }^{136}$.

McCloy und seine Mitarbeiter taten ihr Bestes, um eine solche Entwicklung zu verhindern. Demonstrative Erklärungen bei verschiedenen Anlässen sollten Zweifel an der amerikanischen Entschlossenheit zur uneingeschränkten Durchführung des Rückerstattungsprogramms zerstreuen. So trat im Dezember 1949 das Amt des amerikanischen Hohen Kommissars öffentlich und demonstrativ den Gerüchten über eine Änderung der amerikanischen Rückerstattungspolitik entgegen ${ }^{137}$, nachdem auch in den USA bereits besorgte Stimmen laut geworden waren ${ }^{138}$. Großes Aufsehen erregte McCloys Rede in Stuttgart anläßlich der Eröffnung des Amerikahauses am 6. Februar 1950, in der er nicht nur die allgemeinen aktuellen Grundlinien der amerikanischen Deutschlandpolitik darlegte, sondern auch explizit auf Fragen der Wiedergutmachung einging. McCloy zufolge entsprach es „weiterhin der amerikanischen Politik, allen Personen und Organisationen, die durch rassische oder politische Diskriminierung während der Nazizeit ihres Eigentums beraubt wurden, dieses Eigentum zurückzuerstatten oder ihnen eine adäquate Entschädigung zukommen zu lassen. Es entspricht der amerikanischen Politik, daß alle Personen, die aus rassischen, ideologischen oder religiösen Gründen von den Nazis verfolgt wurden und körperliche Schäden oder Verletzungen erlitten, entschädigt werden ... Ein Wiederaufbau ohne Rücksicht auf diese Verpflichtungen würde verkehrt sein und ein Omen für ein zukünftiges Unglück darstellen. ${ }^{139}$ Hierbei bezog er sich ausdrücklich auf offizielle Anweisungen, die ihm bei seinem jüngsten Besuch in Washington mitgegeben worden waren.

Wenige Monate später erneuerte McCloy diese Erklärungen anläßlich der Eröffnung des Amerikahauses in Hannover. Dabei tadelte er insbesondere die zögerliche deutsche Haltung bei der Rückerstattung und hob hervor, daß der "Geist und die Schnelligkeit,

133 Ritter von Lex (Ministerialdirigent im bayer. Innenministerium) an bayerisches Justizß̂̀inisterium, 25.6. 1948, BayHStA, MInn 79666.

134 Raymond an OMGUS, Administration of Justice Branch, 8. 1. 1949, IfZ-Archiv, MF 260, OMGUS-LD 17/ 214-2/8.

135 Zum Anteil der einzelnen Verfahrensstufen und der Vergleiche vgl. Schwarz, Rückerstattung nach den Gesetzen der Alliierten Mächte, S. 349-356.

136 McLain an Executive Secretary, (ca.Nov./Dez.1949), IfZ-Archiv, MF 260, OMGUS-LD 17/200-2/8.

137 Office of the U.S. High Commissioner for Germany, Press Release, 19. 12. 1949, USNA, RG 59, 262.0041, Box 1041 .

138 Siehe z.B. Bruno Weil an HICOG, 17. 11. 1949, IfZ-Archiv, MF 260, OMGUS-LD 17/200-2/8.

139 Ansprache McCloys in Stuttgart am 6.2. 1950, in: John J. McCloys Reden zu Deutschland- und Berlinfragen. Publizistische Aktivitäten und Ansprachen des Amerikanischen Hochkommissars für Deutschland 1949-1952, hrsg. v. Erika J. Fischer u. Heinz D. Fischer, Berlin 1986, S.67. 
mit der es (sci. das deutsche Volk) ihr nachkommt, ... in beträchtlichem Ausmaße die Haltung der anderen Völker beeinflussen" werde ${ }^{140}$. Auch bei späteren Gelegenheiten drängten der amerikanische Hohe Kommissar und sein Stab auf die beschleunigte Abwicklung der Rückerstattung ${ }^{141}$, und McCloy nutzte weiterhin jede Gelegenheit, die Deutschen darauf hinzuweisen, daß eine Abschwächung der Rückerstattung nicht in Frage komme ${ }^{142}$.

Zugleich drängten sie wiederholt auf konkrete Schritte zur schnelleren Abwicklung der Rückerstattungsverfahren. Zu diesem Zwecke fanden regelmäßige Besprechungen mit den zuständigen deutschen Stellen statt, bei denen auf das Bearbeitungstempo gedrückt wurde. McCloy selbst trat mehrfach an die Ministerpräsidenten der US-Zone heran, um geeignete Maßnahmen zur Beschleunigung des Rückerstattungsprogramms anzumahnen. So wies er etwa den württemberg-badischen und den bayerischen Ministerpräsidenten im Januar 1950 darauf hin, daß zur Einhaltung der avisierten Frist bis Ende 1951 eine Verdrei- bzw. Vervierfachung des personellen und materiellen Aufwandes dringend erforderlich sei ${ }^{143}$. Die Entwicklung der Bearbeitungszahlen sowie die Verwirklichung der geforderten Maßnahmen wurde ständig überprüft, wobei die Angesprochenen den Wünschen des Hohen Kommissars freilich nur zum Teil nachkamen ${ }^{144}$. Sowohl finanzielle Schwierigkeiten als auch das bereits erwähnte Problem, genügend unbelastete Juristen für dieses Gebiet zu finden, machten die Erfüllung dieser Wünsche selbst bei gutem Willen schwierig ${ }^{145}$.

\section{Die JRSO im Konflikt mit den jüdischen Gemeinden und den Ländern}

Einer der zentralen Streitpunkte bei der Entstehung des Rückerstattungsgesetzes war die Frage der jüdischen Nachfolgeorganisationen gewesen. Das gemeinsame Komitee der amerikanischen jüdischen Organisationen, das auch entscheidenden Anteil an der Ausgestaltung dieses Gesetzes hatte, begann bereits im Frühjahr 1947 mit den Vorbereitungen für eine solche jüdische Nachfolgeorganisation, wobei es sich vor allem auf die durch den amerikanischen Militärgouverneur Lucius D. Clay gegebenen Zusagen stützte. Im Mai 1947 kam es zur Gründung der "Jewish Restitution Commission“ (JRC) mit Sitz in New York. Um die geforderte Repräsentativität zu erreichen, wurden eine ganze Reihe weiterer amerikanischer und außeramerikanischer jüdischer Organisationen aufgenommen, darunter auch die „Interessensvertretung Israelitischer Kultusgemeinden “ in der US-Zone, die sich durch Philipp Auerbach vertreten ließ ${ }^{146}$. Im No-

140 Ansprache McCloys in Hannover am 22.5. 1950 vor Kulturpolitikern, in: ebenda, S. $89 \mathrm{f}$.

141 Siehe dazu Minutes of Meeting Held on November 7, 1950, Headquarters Building, Frankfurt, between the U.S. High Commissioner and the Ministerpresidents of the Four Laender in the U.S Zone, WNRC, RG 466, McCloy Papers, Box 21: „McCloy expressed concern about the tendency among the courts, particularly in Bavaria, to drag their feet in restitution cases, apparently on the assumption that control of a restitution program will be relinquished by the Allies in the next future."

142 Vgl. Neue Zeitung, 10.10. 1950, „Freiheit und Frieden nur durch gemeinsame Anstrengungen“ (Text der Rundfunkansprache McCloys am 8.10.); McCloy an die Ministerpräsidenten der US-Zone, 11.6. 1951, abgedruckt in: Bayerischer Landtag, 2. Wp. 1950-1954, Drucksachen, Beilage 1138.

143 McCloy an R. Maier, 24. 1. 1950, BadWürtHStA, EA 1/90, Bü. 709; McCloy an Ehard, 23. 1. 1950, BayMJ, 1101a, H. 7.

144 Siehe etwa Charles P. Gross an R. Maier, 2.6. 1950 u. 18.7. 1950, BadWürtHStA, EA 1/90, Bü. 709.

145 Siehe u. a. Konrad (Staatssekretär im bayer. Justizministerium) an Hans Ehard, 3. 2. 1950, BayMJ, 1101a, H. 7; Küster an R. Maier, 24.2. 1950, BadWürtHStA, EA 1/90, Bü. 709.

${ }^{146}$ Irwin S. Mason an W.C. Haraldson, 30.1. 1948, IfZ-Archiv, MF 260, OMGUS, POLAD 793/46. Vgl. auch 
vember, kurz vor der Verabschiedung des Rückerstattungsgesetzes, wandte sich ihr Präsident Edward M. Warburg schließlich an das State Department mit der Bitte um Anerkennung der JRC als jüdische Nachfolgeorganisation im Rahmen des Militärregierungsgesetzes Nr. $59^{147}$.

Die Absicht des State Departments, diesem Wunsch nachzugeben ${ }^{148}$, traf allerdings auf den Widerstand des War Departments. Major General Daniel Noce, der dort Chef der Civil Administration war, teilte dem State Department am 23. Dezember die Auffassung seines Ministeriums mit, „daß eine ausländische oder nichtdeutsche Organisation nicht zur Nachfolgeorganisation für erbenloses Eigentum unter Militärregierungsgesetz Nr.59 ernannt werden sollte, und daß erbenloses Eigentum von Verfolgten innerhalb Deutschlands für die Hilfe und Rehabilitierung von Personen der selben Herkunft wie die früheren Besitzer verwendet werden sollte ${ }^{\text {“149. }}$. Angesichts der Tatsache, daß die Amerikaner die alliierten Verhandlungen im Kontrollrat über ein gemeinsames Rückerstattungsgesetz hauptsächlich wegen der Forderung nach einer konfessionellen, nichtdeutschen Nachfolgeorganisation hatten scheitern lassen, war dies eher verwunderlich.

Die amerikanische Militärregierung in Deutschland drängte dagegen nunmehr auf eine schnelle Entscheidung, hatte man doch die Anmeldefrist für die Rückerstattungsansprüche reichlich knapp bemessen: Nur Anmeldungen bis zum 31. Dezember 1948 waren gültig. Die restriktive Ausschlußfrist diente dem Zweck, so rasch als möglich für Rechtssicherheit auf diesem Gebiet zu sorgen. Nachdem Clay dem War Department bedeutet hatte, daß in der Frage einer jüdischen Nachfolgeorganisation bindende Absprachen mit den jüdischen Organisationen bestanden, von denen man nicht ohne Gesichtsverlust Abstand nehmen könne ${ }^{150}$, erkannte Washington schließlich Anfang April die Jewish Restitution Commission formell an ${ }^{151}$. Allerdings änderte sie auf Wunsch der amerikanischen Militärregierung ihre Bezeichnung in Jewish Restitution Successor Organization (JRSO) - dies sollte dem Eindruck entgegenwirken, daß es sich hier um eine regierungsamtliche Einrichtung handle ${ }^{152}$.

Durch diese Querelen ging kostbare Zeit verloren, weshalb die jüdischen Organisationen versucht hatten, die Arbeit schon vor der offiziellen Anerkennung der JRSO aufzunehmen. Sie entsandten Repräsentanten in die US-Zone, die mit dem Aufspüren des erbenlosen Eigentums beauftragt waren. Neben den Schwierigkeiten, die sich aus der Quasi-Illegalität ihrer Tätigkeit ergaben, machte ihnen dabei insbesondere die häufig ausgesprochen unfreundliche Haltung zu schaffen, mit der sie von den Vertretern der deutschen jüdischen Gemeinden empfangen wurden ${ }^{153}$. Diese sahen ihre eigenen

Ernest H. Weisman, Die Nachfolge-Organisationen, in: Das Bundesrückerstattungsgesetz, von Friedrich Biella u.a., München 1981, S. 728.

147 Edward M. Warburg an Secretary of State, 3.11. 1947, USNA, WDCAD, RG 165, Box 358.

148 Entwürfe für Genehmigungsschreiben von Capt. Hemmendinger (Dept. of State) v. 26.11. u. 2.12. 1947, USNA, WDCAD, RG 165, Box 358.

149 Daniel Noce an Charles E. Saltzman (Assistant Secretary of State), 23. 12. 1947, USNA, WDCAD, RG 165, Box 358.

150 Clay an Noce, 7.2. 1948, IfZ-Archiv, MF 260, OMGUS, POLAD 793/46.

151 War Department, CSCAD, an OMGUS, 3.4. 1948, IfZ-Archiv, MF 260, OMGUS, AG 1948/184/1.

152 Hays an Chief of Staff US Army for CSCAD, 31.1. 1948, IfZ-Archiv, MF 260, OMGUS, POLAD 793/46.

153 Siehe etwa den geharnischten Brief Werner Peisers (Restitution Consultant des AJDC) vom 30.11. 1947 an den Vorsitzenden der Israelitischen Kultusgemeinde Württemberg Josef Warscher, in dem er sich über den Hinauswurf eines von ihm beauftragten Vertreters beschwert: „What's on your mind? Since when can a German Jewish community send home an employee of an American Palestine organization backed up by 
Interessen am Eigentum der früheren jüdischen Gemeinden durch die JRSO bedroht, die gleichfalls große Teile dieser Werte für sich reklamierte und durch die Ausführungsverordnung Nr. 3 zum Militärregierungsgesetz Nr. 59 in die Nachfolgeschaft des ehemaligen Gemeindevermögens eingesetzt wurde ${ }^{154}$. In der Tat konnten die jüdischen Nachkriegsgemeinden in Deutschland sowohl zahlenmäßig als auch in ihrer Zusammensetzung nur bedingt als Nachfolger der früheren jüdischen Gemeinden gelten ${ }^{155}$. Im Februar 1948 klagte der vom American Joint zur Vorbereitung der Arbeit der JRSO befaßte Werner Peiser seiner vorgesetzten Stelle in Paris: „The more things advance the more obvious becomes the attitude of the German Jewish Communities which strive to eliminate the Successor Organization. “156 Tatsächlich hätte sich zu diesem Zeitpunkt mit einiger Berechtigung auch das Gegenteil behaupten lassen können. Insbesondere Philipp Auerbach geriet über diese Fragen in heftige Konflikte mit der JRSO, der er, wie gesagt, selbst als Vertreter der jüdischen Gemeinden in der US-Zone angehörte, weshalb sie längere Zeit nach einem Weg suchte, ihn loszuwerden ${ }^{157}$.

Doch wurden die Ansprüche der JRSO auch von anderer Seite bedroht. Im Dezember 1948 beantragte das Polnische Rote Kreuz, als Nachfolgeorganisation für das erbenlose Eigentum polnischer Staatsangehöriger in der US-Zone anerkannt zu werden. Die Ansprüche überschnitten sich dabei zum großen Teil mit denen der JRSO. Die amerikanische Militärregierung zögerte deshalb, dem Polnischen Roten Kreuz den gewünschten Status zu verleihen, fürchtete sie doch, daß scheinbar geklärte Fragen wieder neu gestellt werden könnten und dies letztendlich in eine Kritik ihrer Politik münden würde, die der JRSO als einer amerikanischen Gesellschaft das Recht, ehemaliges deutsches Eigentum zu beanspruchen und aus Deutschland fortzuführen, zugestand: „It is desirable to avoid further controversy on this subject. ${ }^{\text { } 158}$ Washington vermerkte dazu kategorisch, daß der Antrag des Polnischen Roten Kreuzes auf Anerkennung als Nachfolgeorganisation „abgelehnt werden solle, und daß die JRSO alles Eigentum polnischer Staatsbürger erhalten solle, die unter die in ihrer Ernennung geregelte Zuständigkeit fielen. Es besteht kein rechtliches Hindernis. " ${ }^{159}$ Der Kalte Krieg und die Verpflichtung gegenüber der JRSO als einer amerikanischen Organisation dürften die Überwindung der eventuell vorhandenen Bedenken erleichtert haben.

Auch Versuche von gewerkschaftlicher Seite, als Nachfolgeorganisationen für erbenloses Gewerkschaftsvermögen ernannt zu werden, hatten keinen Erfolg. Das heißt freilich nicht, daß die Gewerkschaften keine Möglichkeit gehabt hätten, derartiges Vermögen zu beanspruchen; sie mußten allerdings jeweils individuell den Nachweis

more than 5.000.000 Jews?“, IfZ-Archiv, MF 260, OMGUS-CAD 17/261-2/1. Vgl. auch Weisman, Nachfolge-Organisationen, S. $785 \mathrm{f}$.

154 Ausführungs-Verordnung Nr.3 zum MRG 59, Amtsblatt der Militärregierung Deutschland, Amerikanisches Kontrollgebiet, Ausgabe J, 1.8. 1948, S. 3-5.

155 Vgl. Ben Ephraim, Der steile Weg zur Wiedergutmachung, in: Die Juden in Deutschland, 1958/59 - 5719, Ein Almanach, hrsg.v. Heinz Ganther, Hamburg 1959, S. 296; Harry Maòr, Über den Wiederaufbau der jüdischen Gemeinden in Deutschland seit 1945, Mainz 1961, S. $153 \mathrm{f}$.

156 Peiser an Georg Weis (AJDC, Paris), 11.2. 1948, IfZ-Archiv, MF 260, OMGUS-CAD 17/261-2/1.

157 Jerome J. Jacobson (AJDC, Paris) an Eli Rock (AJDC, New York), 14. 10. 1949, Anlage: Memorandum von Benjamin Ferencz an Moses Leavitt, YIVO-Archiv, RG 347, AJC, Records FAD-1, Box 36; Nehemiah Robinson an Hevesi u. Rock, 13.11. 1950, ebenda.

158 Hays an Department of the Army/CSCAD, for Lynch, 18. 12. 1948, IfZ-Archiv, MF 260, OMGUS, AG 1949/9/5.

159 Department of the Army from CSCAD an OMGUS, 18.12. 1948, IfZ-Archiv, MF 260, OMGUS, AG 1949/ $9 / 5$. 
führen, daß sie als Nachfolger für das betreffende Vermögen in Frage kamen ${ }^{160}$. So blieb die JRSO schießlich im Bereich der amerikanischen Besatzungszone die einzige Nachfolgeorganisation für erbenloses Eigentum.

Anfang 1950 saß die JRSO in der US-Zone auf einem Berg von etwa 150.000 unerledigten Ansprüchen, von denen nach ihrer Schätzung abzüglich der ungültigen Forderungen etwa 50.000 bis 75.000 Fälle übrig bleiben würden ${ }^{161}$. Dabei sah sich die JRSO enormen Problemen gegenüber, die nicht nur aus dem Widerstand der Rückerstattungspflichtigen, sondern auch aus erheblichen innerjüdischen Konflikten resultierten. Insbesondere die Tatsache, daß das Gutachten Nr.1 des Nürnberger Appellationsgerichtshofes der JRSO auch den Anspruch auf verspätet eingereichte Individualansprüche zugesprochen hatte, führte zu heftigen Protesten der Betroffenen. Allerdings war die JRSO dann bemüht, in einem „Board of Equity”-Verfahren die daraus entstehenden Härten soweit als möglich abzufedern ${ }^{162}$. So war jedenfalls bis zu diesem Zeitpunkt kein einziger JRSO-Fall durch eine Rückerstattungskammer entschieden ${ }^{163}$. Das eigentliche Ziel der JRSO, nämlich so schnell als möglich Geld aus Deutschland für die Rehabilitierung jüdischer Opfer des Nationalsozialismus im Ausland zu erlangen, war unter diesen Bedingungen in weite Ferne gerückt.

Deshalb traten im Februar 1950 Edward M. Warburg und Nahum Goldmann in New York an McCloy heran und unterbreiteten ihm den Vorschlag, die offenen Rückerstattungsverfahren der JRSO gegen die Zahlung einer Pauschalsumme an die Länder der US-Zone abzutreten. In einem nachgereichten Memorandum machten sie ihm dies unter anderem damit schmackhaft, daß durch derartige Globalabkommen mit den Ländern mehr als die Hälfte der Fälle sofort erledigt würde. Der Vorschlag sei ebenso nützlich für die deutsche Wirtschaft und politische Situation als auch für die Verwirklichung der Ziele der amerikanischen Rückerstattungspolitik ${ }^{164}$. Tatsächlich war McCloy von der vorgeschlagenen Globalregelung angetan, und als er sich am 10. April mit Nahum Goldmann (Jewish Agency), Maurice Boukstein (Jewish Agency), Joseph Schwartz (AJDC) und Benjamin B. Ferencz (JRSO) traf, zeigte er sich außerordentlich entgegenkommend. Zur Zufriedenheit der jüdischen Vertreter erklärte er sich bereit, die Angelegenheit den Ministerpräsidenten der US-Zone nahezubringen, wobei es allerdings „ein schwieriges Problem sei, hier Druck auf die Deutschen auszuüben“165. Die Grundidee - schleunige Abwicklung der individuellen Verfahren durch globale Ablösung - erschien ihm geradezu als das Ei des Kolumbus, so daß er nachfragte, ob Ähnliches nicht auch bei der Entschädigung machbar sei. Die Attraktivität dieses Gedankens für McCloy läßt sich damit erklären, daß er hervorragend geeignet schien, zwei gegensätzliche Intentionen seiner Politik zu versöhnen: Einmal strikte Durchführung der Rückerstattung und zum anderen die innen- und außenpolitische Stabilisierung der Bundesrepublik.

160 Hays an OMG Bremen, Manpower Advisor and Property Control Branch, 20. 12. 1948, IfZ-Archiv, MF 260, OMGUS-LD 17/214-2/9.

161 Warburg u. Goldmann an McCloy, 20.3. 1950, anbei Memorandum: „The overall settlement of JRSO restitution claims in the American Zone of Germany“, WNRC, RG 466, McCloy Papers, Box 7.

162 Vgl. Ben Ephraim, Der steile Weg zur Wiedergutmachung, S. 303-306; Weisman, Nachfolge-Organisationen, S. $751 \mathrm{f} ., 765 \mathrm{ff}$.

163 Warburg u. Goldmann an McCloy, 20.3. 1950 (Anm. 161).

164 Ebenda.

165 Minutes of Meeting with McCloy, 10.4. 1950, WNRC, RG 466, McCloy Papers, Box 12. 
Schon am nächsten Tag nutzte McCloy die Gelegenheit einer Besprechung mit den Ministerpräsidenten der US-Zone, Hans Ehard (Bayern), Christian Stock (Hessen), Reinhold Maier (Württemberg-Baden) und Wilhelm Kaisen (Bremen), ihnen den Vorschlag eines Globalabkommens mit der JRSO ans Herz zu legen ${ }^{166}$. In der Folge nahmen die Länder alsbald Verhandlungen mit der JRSO auf, wobei HICOG im Hintergrund präsent blieb und mehrfach Treffen organisierte, um den Fortgang der Verhandlungen zu beschleunigen ${ }^{167}$. Doch gerieten die im April aufgenommenen Gespräche zwischen der JRSO und den deutschen Landesvertretern bald ins Stocken, weshalb Ferencz, der die JRSO-Delegation leitete, heftig über die Verschleppungstaktik der Länder klagte. Der stellvertretende amerikanische Hohe Kommissar Buttenwieser stimmte dieser Einschätzung zu, verwies aber darauf, „daß HICOG über keine Mittel verfüge, die Deutschen zur Erfüllung dieser Forderung zu zwingen “ ${ }^{168}$. Zwingen im Sinne einer direkten Kontrolle konnten McCloy und seine Mitarbeiter die Deutschen freilich nicht, doch übten sie auch weiterhin hartnäckig Druck auf verschiedenen Ebenen aus.

Als McCloy am 22. August wieder mit den Ministerpräsidenten der US-Zone zusammentraf, las er ihnen die Leviten: Das Abkommen sei als Voraussetzung für die internationale Gleichberechtigung Deutschlands unbedingt erforderlich, die Länder würden jedoch die Verhandlungen blockieren. Er warnte die Ministerpräsidenten dringend davor, auf Erleichterungen der alliierten Vorbehaltsrechte im Bereich der Rückerstattung zu spekulieren: „As long as he had anything to say he would see to it that this reserve power were kept until these just claims of the JRSO were settled satisfactorily and if the Laender governments wouldn't agree to a global' settlement, then each individual claim would be prosecuted through all the courts, even if it were to take years" ${ }^{169}$. Letzteres hätte er in Wahrheit allerdings nur zu gerne vermieden.

Drei Tage später brachte HICOG die beiden Parteien in ihrem Hauptquartier in Frankfurt erneut zusammen. Alle Länder erklärten dabei ihre prinzipielle Bereitschaft zu einem Abkommen, machten aber eine Reihe von Schwierigkeiten geltend. So seien die von der JRSO angegebenen Zahlen über die in den Vergleich einzubeziehenden Werte vielfach ungenau oder überhöht, zudem sei die Finanzierung dieses Abkommens ein großes Problem, hier dürfe man auch die Landtage nicht übergehen ${ }^{170}$. Ein wesentliches Hindernis für eine Einigung sei aber auch, daß die „Länder vor die außerordentlich schwierige Aufgabe gestellt (seien), die seitens der JRSO abzutretenden Ansprüche auch gegenüber gutgläubigen Rückerstattungspflichtigen durchzusetzen, was nach deutschem Recht wenig Aussicht auf Erfolg “ ${ }^{171}$ habe.

Umgekehrt argumentierte die JRSO gegenüber den Ländern gerade damit, daß diese gegenüber ihren Staatsbürgern kulanter vorgehen könnten, als es der JRSO möglich sei. Zur Taktik der JRSO gehörte also primär das "Vorzeigen der Instrumente“ - das hieß, die Drohung mit der unnachgiebigen Realisierung ihrer Rückerstattungsansprüche in

166 Minutes of Meeting between the U.S. High Commissioner and the Ministerpresidents of the four Laender in the U.S. Zone, 11.4. 1950, WNRC, RG 466, McCloy Papers, Box 12.

167 McCloy an die Ministerpräsidenten der US-Zone, 8.5. 1950, WNRC, RG 466, McCloy Papers, Box 12.

168 Zachariah Shuster an John Slawson, 11.8. 1950, YIVO-Archiv, RG 347, AJC, FAD-1, Box 27.

169 Minutes of Meeting between the U.S. High Commissioner and the Ministerpresidents of the four Laender in the U.S. Zone, 22.8. 1950, WNRC, RG 466, McCloy Papers, Box 18.

170 Protokoll über die Sitzung am 25.8. 1950 in Frankfurt/M., betr. die Durchführung einer Global-Vereinbarung zur Regelung von Rechtsansprüchen der JRSO aus dem MRG 59, BayHStA, MA 114243.

171 W. Hilpert an Shepard Stone, 22.9. 1950, BadWürtHStA, EA 1/920, Bü. 715. 
eigener Regie. Gleichzeitig wurde aber auch die große außenpolitische Bedeutung einer Einigung hervorgehoben, und wenn dies nicht ausreichend schien, wurde auch noch mit dem amerikanischen Hohen Kommissar gedroht, der dieses Spiel bereitwillig mitmachte. So gelang es der JRSO bald, die Länder auseinanderzudividieren. Am einfachsten war die Situation in Bremen, da es hier nur um ca. 85 strittige Objekte ging. Deshalb wurde bereits am 28. Juni 1951 ein Abkommen unterzeichnet, wonach die JRSO 1,5 Mio. DM erhalten sollte ${ }^{172}$.

Hessen hingegen hatte es mit Ansprüchen in ganz anderer Größenordnung zu tun. Aber auch hier war bereits gegen Ende des Jahres ein Abkommen in Sicht, das der JRSO 25 Mio. DM zusprach. Grundlage der Berechnung war (wie bei allen diesen Abkommen) nicht der tatsächliche Wert der entzogenen Gegenstände, sondern eine Art Konkursquote, die auf der durchschnittlichen Höhe der bislang erreichten Vergleiche beruhte. Deshalb war das Abkommen, wie Finanzminister Werner Hilpert gegenüber dem hessischen Kabinett erläuterte, „in finanzieller Hinsicht ... für das Land auch insofern vorteilhaft, als die vorgeschlagene Abfindungssumme weit unter dem tatsächlichen Wert der abzutretenden Ansprüche liegt." Zudem könne das Land nunmehr Härten ausgleichen, „die sich aus der Durchführung des Mil-Reg. Gesetzes Nr. 59 ergeben haben und das deutsche Wirtschaftsleben in erheblichem Maße belasten " ${ }^{173}$. Am 13. Februar 1951 gab der Hessische Landtag seine Zustimmung. Während die New York Times die Bescheidenheit des Abkommens hervorhob, das nur einen Bruchteil der tatsächlich entzogenen Werte bezahlte ${ }^{174}$, kritisierte die Frankfurter Allgemeine Zeitung das Abkommen scharf, da hier die Wiedergutmachung zu einem Geschäft gemacht würde ${ }^{175}$.

Mit Besorgnis betrachteten Württemberg-Baden und Bayern dieses Abkommen, das in ihren Augen die Verhandlungsfront schwächte ${ }^{176}$. Einer der schärfsten Gegner eines Globalabkommens mit der JRSO war Otto Küster. Die Globalisierung der Rückerstattungsforderungen war für ihn gleichbedeutend mit einer Kommerzialisierung der Wiedergutmachung, die deren moralische Grundlagen verdunkle ${ }^{177}$. Beschwörend schrieb Küster am 13. Februar 1951 an Ministerpräsidenten Reinhold Maier: „... eine von der Aktiv- wie von der Passivseite her so umstrittene Masse zu erwerben wäre ein verhängnisvoller Akt. Den Juden selbst und einer jüdischen Organisation verzeiht man allenfalls, daß sie ihre Ansprüche durchfechten. Der beamtenmäßige Prozeßbetrieb eines deutschen Fiskus, der die Ansprüche aufgekauft hat, wird dagegen notwendig $\mathrm{Haß}$ und Verachtung auf den Staat ziehen." Besonders verwerflich empfand Küster den Vorschlag der JRSO, quasi zur Tarnung eine GmbH vorzuschieben, „die dann das mit Rabatt gekaufte, dem wahren Berechtigten vorenthaltene Judengut privatkapitalistisch“ verwerten könne ${ }^{178}$.

Daß die jüdischen Organisationen hier verhältnismäßig unbesorgt um die politisch-

${ }^{172}$ Vgl. Weisman, Nachfolge-Organisationen, S. 769. Die effektive Abfindungssumme verringerte sich schließlich noch auf 1,25 Mio. DM.

${ }^{173}$ Kabinettsvorlage des hessischen Finanzministers Hilpert, 24. 11. 1950, HessHStA, Abt. 502, Nr. 2008.

174 Jack Raymond, nJews' Claims Cut to Aid Restitution. Fears of German Sovereignity Rise Spur Bulk Settlement with Individual States", New York Times, 13.2. 1951.

$175 \mathrm{Vgl}$. Frankfurter Allgemeine Zeitung, 28. 2. 1951, „Fragwürdige Ablösung“.

176 Küster an R. Maier, 1.3. 1951, BadWürtHStA, EA 1/920, Bü. 715.

177 Referat Küsters vor der Arbeitstagung jüdischer Juristen in Düsseldorf, in: Staatsanzeiger für WürttembergBaden, 8.3. 1952.

${ }^{178}$ Küster an R. Maier, 13.2. 1951, BadWürtHStA, EA 1/920, Bü. 715. 
moralische Wirkung ihrer Forderungen auftraten, hatte seinen Grund darin, daß sie wenig an inneren Vorgängen in Deutschland interessiert waren. Im Gegensatz zu Otto Küster ging es ihnen zumindest zu diesem Zeitpunkt nicht primär um die Verbesserung des deutsch-jüdischen Verhältnisses, sondern darum, schleunigst Mittel für die Betreuung notleidender jüdischer Opfer des Nationalsozialismus aufzubringen - die überwiegend außerhalb Deutschlands lebten. Küster, der dadurch seine eigenen Bemühungen entwertet sah, schrieb deshalb verzweifelt: „Haben für solche jammervollen Projekte die deutschen Wiedergutmachungswilligen Jahr um Jahr geschrieben, geworben und gestritten? Haben dafür unsere jüdischen Mitbürger gelitten?“ ${ }^{179}$

Doch auch Württemberg-Baden trat schließlich im März in Verhandlungen ein ${ }^{180}$, nachdem zwischenzeitig unter anderem der US-Landeskommissar Charles P. Gross heftig gedrängt hatte, dem hessischen Beispiel zu folgen ${ }^{181}$. Allerdings fand man hier eine etwas veränderte Geschäftsgrundlage für ein Abkommen mit der JRSO. Küster setzte sich wenigstens insoweit durch, als nicht die noch offenen Rückerstattungsprozesse der JRSO übernommen wurden. Statt dessen wurde ein „Plan erwogen, der der JRSO auf Kosten des Landes gewisse Mittel in die Hand geben würde, ohne daß das Land gegenüber Privatpersonen in die Rolle eines Rückerstattungsgläubigers gerät“ ${ }^{182}$. So gründete man das Abkommen auf Grundstücke, die bereits im Eigentum der JRSO waren bzw. solche, die durch das Reich oder eine NS-Organisation entzogen worden waren, sowie auf Rückerstattungsansprüche, die sich aus der Ablieferungspflicht von Edelmetallen und Schmuck usw. an Pfandleihanstalten ergaben. Dazu kamen Ansprüche gegen Kreditinstitute aus der Entziehung von Wertpapieren sowie gegen das Deutsche Reich aus der Entziehung von Wertpapieren, Hypotheken, Bankguthaben etc. und schließlich solche, die auf der Zerstörung von Friedhöfen und Synagogen beruhten ${ }^{183}$.

Das Ansinnen, für diese Zwecke einen US-Kredit zu erhalten, brachte allerdings eine schroffe Abfuhr des amerikanischen Landkommisars ein: „there is no money available for the purpose you suggest. ${ }^{184}$ Nach langem Hin und Her über die endgültige Höhe der Abfindung und die Zahlungsbedingungen wurde schließlich am 29. November 1951 vorbehaltlich der Genehmigung durch den Landtag ein Abkommen in Höhe von 10 Mio. DM zwischen Württemberg-Baden und der JRSO geschlossen. Am 12. Dezember stimmten alle Landtags-Fraktionen einmütig für die Annahme - mit Ausnahme der fünf Abgeordneten der Deutschen Gemeinschaft/Block der Heimatvertriebenen und Entrechteten (DG/BHE) ${ }^{185}$, die sich in erster Linie den Vertriebenen verpflichtet fühlten.

Am längsten zog sich die Einigung mit Bayern hin. Dazu trug auch Philipp Auerbach bei, der sich in dieser Frage gegen die JRSO stellte und deshalb ein „schmerzliches Problem für die jüdischen Organisationen darstellte", wie Zachariah Shuster vom American Jewish Committee im Januar 1951 dem bayerischen US-Landeskommissar Geor-

179 Otto Küster, „Verhängnisvolles Ansinnen“, Deutsche Zeitung, 12.2. 1951.

180 Besprechung des württemberg-badischen Finanz- und Justizministeriums mit Vertretern der JRSO, 27.3. 1951, BadWürtHStA, EA 1/920, Bü. 715.

181 Gross an R. Maier, 14.2. 1951, BadWürtHStA, EA 1/920, Bü. 715.

182 Küster an Beyerle, 20.6. 1951, BadWürtHStA, EA 1/920, Bü. 715.

183 Landtag von Württemberg-Baden, 2. Wp. 1950-1952, 44. Sitzung am 12.12. 1951, Stenographische Berichte, S. $1705 \mathrm{f}$.

184 H.M. Coverley (Acting Land Commissioner/Württ.-Baden) an R. Maier, 28.6. 1951, BadWürtHStA, EA I/ 920, Bü. 715 .

185 Landtag von Württemberg-Baden, 2.Wp. 1950-1952, 44. Sitzung vom 12. 12.1951, Stenographische Berichte, S. 1705-1709. 
ge N. Shuster erklärte ${ }^{186}$. Doch am 10. März 1951 wurde, wie gesagt, Auerbach verhaftet $^{187}$, wodurch einer der hartnäckigsten Gegner des Abkommens ausgeschaltet war. Ende des Monats teilte schließlich Ministerpräsident Ehard, der noch kurz zuvor im Ministerrat die Auffassung vertreten hatte, „daß man jedenfalls noch nicht zu einem Abschluß kommen könne und es notwendig sei, der JRSO einen dilatorischen Bescheid zu geben“ ${ }^{188}$, Finanzminister Rudolf Zorn mit, er „halte ... es doch für notwendig, bald zu einem endgültigen Abschluß zu kommen “ ${ }^{189}$. Dennoch protestierte Ferencz, dessen wiederholte Schreiben gar nicht oder hinhaltend beantwortet worden waren, vier Wochen später bei Ehard: „Es ist für mich unbegreiflich, daß es entweder ihre oder Dr. Zorns Absicht ist, vorsätzlich die Interessen der jüdischen Überlebenden der NaziVerfolgung, die die Almosenempfänger der JRSO sind, mit Füßen zu treten. “190 Daraufhin kam es zu einer Besprechung zwischen Vertretern des bayerischen Staates und der JRSO, in deren gereizter Atmosphäre sich Ehard und Staatssekretär Ringelmann damit rechtfertigten, daß allein die überhöhten Ansprüche der JRSO und die schwierige Haushaltslage des bayerischen Staates bislang eine Lösung verhindert hätten ${ }^{191}$.

$\mathrm{Zu}$ den bayerischen Bedingungen für ein Abkommen gehörten deshalb neben einer drastischen Reduzierung der Forderungen der JRSO vor allem eine ausländische Anleihe ${ }^{192}$. Während Landeskommissar Shuster auf diesen Gedanken freundlich reagiert haben soll ${ }^{193}$, geriet McCloy über einen Brief Ehards, in dem dieser über die schlechte bayerische Finanzlage klagte und deshalb zur Finanzierung eines JRSO-Abkommens einen Auslandskredit forderte, in Rage: Sich nach einem ausländischen Land zur Erledigung dieser Verpflichtungen umzusehen, sei „unrealistisch, wenn nicht gar unmoralisch." McCloy forderte Shuster auf, "persönlich und hart" auf das JRSO-Abkommen zu drängen, da es von „herausragender Bedeutung im Zusammenhang mit jeder konstruktiven Lösung unserer vertraglichen Beziehungen und des neuen politischen Status Deutschlands sei." Zudem werde er sehr zurückhaltend mit weiterer Wirtschaftshilfe für Bayern sein, solange diese Sache nicht bereinigt sei. Zum Abschluß schrieb McCloy: "There are few things more important on our final agenda than this restitution matter, and I wish that you and your staff would concentrate on it, as I am asking the staff in Wuerttemberg-Baden to do likewise. “ 194

Die außergewöhnlich schwierigen Verhandlungen zwischen der JRSO und Bayern zogen sich unter der Leitung Ringelmanns und Ferenczs bis Mitte 1952 hin $^{195}$. Am 10. Juni beschloß der bayerische Ministerrat schließlich, das Abkommen, bei dem man sich auf die Zahlung von 20 Mio. DM geeinigt hatte, dem Landtag zuzuleiten ${ }^{196}$. Hier waren die unerledigten Ansprüche der JRSO wiederum eingeschlossen, denn Ziel auf bayeri-

186 Z. Shuster an Slawson, 9. 1. 1951, YIVO-Archiv, RG 347, AJC, Records FAD-1, Box 27.

187 Vgl. oben, S. 163.

188 Bayerischer Ministerrat, 13.3. 1951, IfZ-Archiv, NL Hoegner, ED 120, Bd. 367.

189 Ehard an Zorn, 30.3. 1951, BayHStA, MA 114243.

190 Ferencz an Ehard, 26.4. 1951, BayHStA, MA 114243.

191 Kiefer (Ministerialdirigent im bayer. Finanzministerium), Aufzeichnung über die Besprechung am 2.5. 1951 mit Vertretern der JRSO in der bayerischen Staatskanzlei, ebenda.

192 Niederschrift der Besprechung am 2.7. 1951 in der bayerischen Staatskanzlei mit Vertretern der JRSO, 2.7. 1951, ebenda.

193 Ringelmann in der Sitzung des bayerischen Ministerrats am 13.3. 195!, IfZ-Archiv, NL Hoegner, ED 120, Bd. 367.

194 McCloy an G.N. Shuster, 24. 7. 1951, WNRC, RG 466, McCloy Papers, Box 29.

195 Siehe dazu die Unterlagen in BayHStA, MA 114243-44.

196 Bayerischer Ministerrat, 10.6. 1952, IfZ-Archiv, NL Hoegner, ED 120, Bd. 372. 
scher Seite war das Ende der Aktivitäten der JRSO auf bayerischem Boden ${ }^{197}$. Nachdem McCloy kurz vor seiner Rückkehr in die Vereinigten Staaten nochmals in dieser Sache interveniert und dazu gedrängt hatte, das Abkommen endlich zu ratifizieren ${ }^{198}$, wurde es am 24. Juli 1952 abschließend vor dem Bayerischen Landtag diskutiert. Sprachen sich die zu dieser Zeit in einer Regierungskoalition stehenden CSU und SPD für das Abkommen aus (erstere allerdings nicht vollständig und mit etlichen Vorbehalten), äußerten Bayernpartei, FDP und BHE hingegen erhebliche Bedenken und votierten gegen das Abkommen bzw. enthielten sich der Stimme. Häufig variiertes Argument war dabei, daß, wie es der Bayernpartei-Abgeordnete August Geislhöringer formulierte, „der Not in Israel eine Not von 10 bis 12 Millionen Deutschen im Osten gegenüberstehe" ${ }^{199}$. Der Bayerische Landtag billigte schließlich das Abkommen „mit Mehrheit gegen eine beachtliche Minderheit und bei einigen Stimmenthaltungen ${ }^{200}$.

Die Länder betrachteten also die im Rahmen der Globalabkommen an die JRSO gezahlten Beträge als eine Art von Lösegeld. Damit wollten sie die von deren Tätigkeit ausgehende wirtschaftliche und politische Beunruhigung beseitigen. Auch das ungewöhnlich starke Engagement McCloys sowie seiner regionalen Vertreter hatte neben moralischen Impulsen v.a. auch den Zweck, die gewünschte Liquidation des Rückerstattungsprogramms zu fördern. Hier handelte es sich in gewisser Weise um eine Politik des Schlußstriches, in welchen Zusammenhang ja auch etwa die zur gleichen Zeit virulente Frage der in Landsberg einsitzenden Kriegsverbrecher fiel ${ }^{201}$. So erscheint McCloys Haltung gegenüber den deutschen historischen Belastungen vordergründig als ambivalent: einerseits eine großzügige Amnestie unter den Landsberg-Häftlingen, andererseits energisches Drängen auf zügige Abwicklung der Rückerstattung sowie den Abschluß von Globalabkommen mit der JRSO. Letzteres hatte - beabsichtigt oder nicht - bedeutsame Nebenwirkungen: Auf dem Wege der Globalisierung solcher Rückerstattungsforderungen trat, wie vor allem von Otto Küster beklagt wurde, eine gewisse Abkehr vom Grundsatz einer restaurativen Wiedergutmachung ein. Doch ging es den Beteiligten hier in erster Linie um praktische Zwecke: innen- und außenpolitische Stabilisierung auf der einen, Mittelbeschaffung für notleidende jüdische Verfolgte im Ausland auf der anderen Seite.

\section{Quantitative Bilanz}

Aufgrund der ausführlichen statistischen Begleitung der Durchführung des Militärregierungsgesetzes Nr. 59 in der US-Zone lassen sich hier auch einige quantitative Ergebnisse der Rückerstattung wiederauffindbaren Vermögens darstellen ${ }^{202}$. Fragt man nach der Zahl der Rückerstattungsfälle, so muß dabei zwischen Individualansprüchen und

197 Zietsch an Ehard, 18. 2. 1952, BayHStA, MA 114244.

198 McCloy an Ehard, 18.7. 1952, ebenda.

199 Bayerischer Landtag, 2. Wp. 1950-1954, 103. Sitzung am 24. Juli 1952, Stenographische Berichte, S. 2644.

200 Ebenda, S. 2649.

201 Vgl. Thomas Alan Schwartz, Die Begnadigung deutscher Kriegsverbrecher. John J. McCloy und die Häftlinge von Landsberg, in: Vierteljahrshefte für Zeitgeschichte 38 (1990), S. 375-414.

202 Vgl. dazu auch Schwarz, Rückerstattung nach den Gesetzen der Alliierten Mächte, S. 345-373.- Bei der Darstellung der Durchführung des Entschädigungsgesetzes der US-Zone wurde in der vorliegenden Arbeit dagegen vor allem deshalb auf eine statistische Auswertung verzichtet, als dieses letztlich nur eine Zwischenstufe zum Bundesentschädigungsgesetz darstellt und man somit keine abschließenden Ergebnisse präsentieren kann. 
JRSO-Ansprüchen unterschieden werden. Die Individualansprüche beliefen sich alles in allem auf ca. $87.000^{203}$. Die JRSO hatte zwar zunächst über 160.000 Ansprüche angemeldet, darunter befanden sich aber zahlreiche Doppelanmeldungen oder solche, die auf bloßen Verdacht hin eingereicht worden waren, so daß die tatsächliche Zahl der Fälle etwa um die Hälfte niedriger $\mathrm{lag}^{204}$.

Das von McCloy formulierte Ziel, die Rückerstattung in der US-Zone bis Ende 1951 abzuschließen, wurde trotz der massiven Interventionen zugunsten einer schleunigen Abwicklung nicht ganz erreicht. Dennoch verlief sie insgesamt gesehen zügig. Waren Ende 1949 gerade erst 14,1 v.H. der individuellen Rückerstattungsansprüche einschließlich der noch nicht bearbeiteten Ansprüche gegen das Deutsche Reich erle$\operatorname{digt}^{205}$, so lag dieser Wert zwei Jahre später immerhin bei 62,2 Prozent. (Für diesen Zeitraum wiesen die Statistiken die Reichsansprüche noch nicht getrennt aus. Ohne diese war die Erledigungsquote noch ein Stück höher.) Nach Ablauf eines weiteren Jahres, Ende 1953, waren bereits 92,7 Prozent dieser Verfahren abgewickelt ${ }^{206}$. Auch die Arbeit der JRSO hatte entsprechende Fortschritte gemacht, wozu vor allem beigetragen hatte, daß bis Anfang 1954 über 56.000 Fälle im Rahmen der Globalabkommen mit den US-Zonen-Ländern geregelt wurden ${ }^{207}$. So konnte ein Vertreter von HICOG zu dieser Zeit feststellen: „It appears to us that for all practical purposes restitution in kind through the Agencies has been completed under U.S. MG Law 59 and that the Restitution Agencies are, for the most part, engaged in the settlement of claims against the Reich. “ ${ }^{208}$ Doch war es zwar „bereits möglich, Urteile gegen das Reich zu erlangen, aber“, wie Bruno Weil Mitte 1951 schrieb, „die materielle Erledigung wird durch den Umstand gehindert, daß es an gesetzlichen Bestimmungen fehlt, durch die die Haftbarmachung des Reichs und der Länder verwirklicht werden könnte “209. Dies betraf beispielweise auch die Wertsachen, die die jüdischen Verfolgten im Februar 1939 abliefern mußten ${ }^{210}$. Die Regelung der auch als „Dritte Masse“ bezeichneten Rückerstattungsansprüche gegen das Deutsche Reich erfolgte erst 1957 durch das Bundesrückerstattungsgesetz ${ }^{211}$.

$\mathrm{Zu}$ welchen Resultaten führte nun die Durchführung des Militärregierungsgesetzes Nr. 59 in der US-Zone? Alles in allem betrug der Wert des dort rückerstatteten wiederauffindbaren Vermögens über eine Milliarde DM. Zählt man die entsprechenden Werte für die britische und französische Zone sowie Westberlin dazu, so gelangt man für die

203 Vgl. ebenda, Tabelle 1: Rechtsanhängige, erledigte und noch anhängige Fälle einschließlich der Reichsansprüche (Ohne Nachfolgeorganisationen), S. 385.

204 McCloy an Acheson, 15.3. 1950, USNA, RG 59, 262.0041/3-1550; Highlights of Property Disposition Activities for Month of June 1950, USNA, RG 59, 262.0041.

205 Summary. Cumulative Statistical Report for Restitution Authorities. 10 November 1947 to 31 December 1949, BayMJ, 1101 a, H. 14.

206 Vgl. Schwarz, Rückerstattung nach den Gesetzen der Alliierten Mächte, Tabelle 2: Rechtshängige, erledigte und noch anhängige Fälle obne Reichsansprüche (Ohne Nachfolgeorganisationen), S. 386. Siehe auch Unterlagen in BayMJ, 1101a, H. 14.

207 Knox Lamb (HICOG/Deputy General Counsel) an Walter Roemer (Bundesjustizministerium), 8. 2. 1954, BayMJ, 1101a, H. 4. Schwarz gibt insgesamt 60.000 auf diesem Wege geregelte Fälle an. Vgl. ders. Rückerstattung nach den Gesetzen der Alliierten Mächte, S. 368.

${ }^{208}$ Lamb an Roemer, 8.2. 1954 (Anm. 207).

209 Bruno Weil, Der Stand der Rückerstattung in der amerikanischen Zone, in: Deutsche Zeitung und Wirtschaftszeitung, 18.8. 1951.

210 Otto Küster, Wiedergutmachung als elementare Rechtsaufgabe, Frankfurt a.M. 1953, S.6.

211 Vgl. dazu Friedrich Biella u. a., Das Bundesrückerstattungsgesetz, München 1981. 
Bundesrepublik auf einen annäherungsweisen Betrag von etwa 3,5 $\mathrm{Mrd}$. $\mathrm{DM}^{212}$. Der Großteil entfiel dabei auf die individuelle Rückerstattung. So waren Ende 1951, als die Rückerstattung wertmäßig etwa zur Hälfte abgewickelt war, etwa $512 \mathrm{Mio}$. DM an individuelle Kläger rückerstattet worden, während die JRSO gerade erst bescheidene 8,7 Mio. DM realisiert hatte ${ }^{213}$. Vor diesem Hintergrund wird auch die Dringlichkeit verständlich, mit der die JRSO die Globalabkommen mit den Ländern der US-Zone, die ihr am Ende insgesamt ca. 47 Mio. DM einbrachten, verfolgte.

Walter Schwarz bezifferte den Gesamtanteil der JRSO an den Ergebnissen der Rückerstattung auf bescheidene acht Prozent ${ }^{214}$, was angesichts des wesentlich höheren Anteils an der Zahl der Gesamtansprüche überrascht. Doch waren die einzelnen Ansprüche von höchst unterschiedlichem Wert. Gerade die "großen Brocken“, d.h. größere Firmen etc., wurden in aller Regel auf dem individuellen Wege zurückerstattet, und dies meist schon in den ersten Jahren. So meldete McCloy im März 1950 nach Washington, daß unter anderem die Kaufhauskette Hertie, Aktien der Deutschen Magnetit AG im Wert von zehn Mio. DM, eine Zellulosefabrik im Wert von sieben Mio. DM und einige andere größere Objekte auf dem Wege des außergerichtlichen Vergleichs rückerstattet worden waren ${ }^{215}$. Der JRSO hingegen blieben meist Ansprüche von geringerem Wert, die zudem häufig stärker umstritten waren. Auch akzeptierte sie im Rahmen der von ihr abgeschlossenen Vergleiche relativ bescheidene Quoten, um so schnell wie möglich an Mittel für ihre caritative Arbeit zu gelangen.

Die historische Bedeutung der JRSO muß jedoch wesentlich höher veranschlagt werden, als aus dem erzielten finanziellen Anteil hervorgeht, sofern man die Angelegenheit unter dem Gesichtspunkt der mit der Rückerstattung einhergehenden gesellschaftlichen Turbulenzen betrachtet. Ihr Auftreten sorgte für erhebliche Beunruhigung unter den Betroffenen und reaktivierte in Deutschland zugleich häufig alte Vorurteile über internationalen jüdischen Kapitalismus. Dies verstärkte sich auch dadurch, daß sich die JRSO-Forderungen weniger auf die großen Arisierungsfälle richteten, als vielmehr neben dem früheren jüdischen Gemeindeeigentum auf die kleineren Fälle, bei denen die Pflichtigen vielfach im Zuge der Rückerstattung selbst in große Schwierigkeiten gerieten. So wurde die JRSO geradezu zum Symbol für den oktroyierten Charakter der Rückerstattung und stand zumindest in den ersten Jahren im Mittelpunkt der Auseinandersetzungen.

Freilich betraf nicht die ganze Rückerstattung wiederauffindbaren Vermögens ausschließlich jüdisches Eigentum, wenngleich es sich dabei um den weitaus größten Teil gehandelt haben dürfte. Exakte Angaben sind allerdings auf der Grundlage der vorhandenen Statistiken nicht möglich. Andere bedeutende Kategorien waren etwa das Vermögen von Parteien, Gewerkschaften, Kirchen und anderen politischen und caritativen Organisationen. Auch in diesem Bereich wurde eine Anzahl von Globalabkommen mit den einzelnen Ländern geschlossen ${ }^{216}$. Die Rückerstattung für diese Gruppen basierte allerdings nicht nur auf dem Militärregierungsgesetz $\mathrm{Nr}$. 59, sondern vor allem auch auf der Kontrollratsdirektive Nr.50. Darin war die Rückgabe des ehemaligen Vermögens

212 Vgl. Schwarz, Rückerstattung nach den Gesetzen der Alliierten Mächte, S. 364.

213 Office of the U.S. High Commissioner for Germany, Office of Economic Affairs, Report for the Month Ending December 31, 1950, USNA, RG 59, 262.0041/1-1751.

214 Vgl. Schwarz, Rückerstattung nach den Gesetzen der Alliierten Mächte, S. 362.

215 McCloy an Acheson, 15.3. 1950, USNA, RG 59, 262.0041/3-1550.

216 Siehe etwa bayerischer Ministerrat, 9.6. u. 20.6. 1953, IfZ-Archiv, NL Hoegner, ED 120, Bd. 376, 378. 
von Parteien, Gewerkschaften, Genossenschaften und sonstigen demokratischen, religiösen und caritativen Organisationen, das sich unter dem im Zuge der alliierten Vermögenskontrolle beschlagnahmten Eigentum der NSDAP und damit zusammenhängender Organisationen befand, geregelt ${ }^{217}$.

Einen Anhaltspunkt über die Verteilung jüdischen und nichtjüdischen Vermögens bei der Rückerstattung gibt die Aufteilung der rückerstatteten Werte nach der Staatsangehörigkeit bzw. dem Wohnsitz der Berechtigten. Nimmt man das Stichjahr 1957, so lebten 44 Prozent der Berechtigten in den USA, 18 Prozent in Deutschland, neun Prozent in Großbritannien, fünf Prozent in der Schweiz, fünf Prozent in Israel und acht Prozent in Mittel- und Südamerika ${ }^{218}$, wobei unter den über 80 Prozent der Berechtigten aus dem Ausland der Großteil jüdischer Herkunft gewesen sein dürfte. In Anbetracht dieses hohen Auslandsanteils stellte es natürlich ein besonderes Problem dar, daß die DM erst von 1953 an schrittweise frei konvertierbar wurde, abgeschlossen war dieser Prozeß erst $1958^{219}$. Der Sonderstatus der JRSO zeigt sich auch daran, daß sie bereits 1951 eine Genehmigung zum Transfer erbenlosen Eigentums ins Ausland erhielt ${ }^{220}$. Der große Anteil der Ansprüche aus den USA, zu denen ja auch noch die JRSO gezählt werden muß, verweist zudem noch einmal auf die Ratio des nachhaltigen und herausragenden Interesses der US-Politik an der Rückerstattung: Hierbei handelte es sich eben auch um ein Stück Wahrung der Vermögensinteressen amerikanischer Staatsbürger.

217 Kontrollratsdirektive Nr.50: Verfügung über Vermögenswerte, die den in der Kontrollratsproklamation Nr. 2 und im Kontrollratsgesetz Nr. 2 aufgeführten Organisationen gehört haben, Amtsblatt des Kontrollrats in Deutschland, Nr. 15, 31.5. 1947, S. 275-278. Vgl. auch Drittes Kapitel, S. $116 \mathrm{f}$.

218 Vgl. Schwarz, Rückerstattung nach den Gesetzen der Alliierten Mächte, S. 366.

219 Vgl. Christoph Buchheim, Die Wiedereingliederung Westdeutschlands in die Weltwirtschaft 1945-1958, München 1990, S. 158-170.

220 Vgl. AJC Contributions to Postwar Economic Rehabilitation of Jewish Victims of Nazi Persecution, September 1965, AJC-Archiv, JSX, Subject Restitution 65-66. 